\title{
Greenhouse Gas Emissions and Removals from Forest Land, Woodlands, and Urban Trees in the United States, 1990-2019
}

\section{Appendix 2.-National Scale Quantitative Uncertainty Estimates for Individual States, 2019}

Appendix 2 (https://doi.org/10.2737/FS-RU-307-Appendix2) in: Domke, Grant M.; Walters, Brian F.; Nowak, David J.; Smith, James, E.; Nichols, Michael C.; Ogle, Stephen M.; Coulston, J.W.; Wirth, T.C. 2021. Greenhouse gas emissions and removals from forest land, woodlands, and urban trees in the United States, 1990-2019. Resource Update FS-307. Madison, WI: U.S. Department of Agriculture, Forest Service, Northern Research Station. 5 p. [plus 2 appendixes]. https://doi.org/10.2737/FS-RU-307.

\begin{tabular}{|l|l|l|l|l|}
\hline \multicolumn{5}{|c|}{ Appendix Contents } \\
\hline Alabama & Alaska & Arizona & Arkansas & California \\
\hline Colorado & Connecticut & Delaware & Florida & Georgia \\
\hline Idaho & Illinois & Indiana & Iowa & Kansas \\
\hline Kentucky & Louisiana & Maine & Maryland & Massachusetts \\
\hline Michigan & Minnesota & Mississippi & Missouri & Montana \\
\hline Nebraska & Nevada & New Hampshire & New Jersey & New Mexico \\
\hline New York & North Carolina & North Dakota & Ohio & Oklahoma \\
\hline Oregon & Pennsylvania & Rhode Island & South Carolina & South Dakota \\
\hline Tennessee & Texas & Utah & Vermont & Virginia \\
\hline Washington & West Virginia & Wisconsin & Wyoming & \\
\hline
\end{tabular}

Appendix Notes:

- Managed forest land for U.S. Territories is not currently included in Section 6.1 Representation of the U.S. Land Base in the "Inventory of U.S. Greenhouse Gas Emissions and Sinks: 1990-2019" (US EPA 2021), therefore U.S. Territories are not included here.

- There is not sufficient Forest Inventory and Analysis data to include estimates of forest ecosystem C stocks, C stock changes, or non- $\mathrm{CO}_{2}$ emissions for the state of Hawaii at this time.

- Not every table is included for every state. This may be due to the resolution of the available data, a lack of observations of the particular land use category or land use conversion category, or there was insufficient data.

- The state-level estimates reported in the following tables will not sum to the national total due to independent rounding. 


\section{Alabama}

Table 1: Quantitative Uncertainty Estimates for Net $\mathrm{CO}_{2}$ Flux from Forest Land Remaining Forest Land (MMT $\mathrm{CO}_{2}$ Eq. and Percent), Alabama

\begin{tabular}{|c|c|c|c|c|c|}
\hline \multirow[b]{2}{*}{ Source } & \multirow[b]{2}{*}{$\begin{array}{l}2019 \text { Flux Estimate } \\
\text { (MMT } \mathrm{CO}_{2} \text { Eq.) }\end{array}$} & $\begin{array}{r}\text { Uncert } \\
(\mathrm{MM}\end{array}$ & $\begin{array}{l}\text { (ange Re } \\
\text { Eq.) }\end{array}$ & to Emiss & timate $^{a}$ \\
\hline & & $\begin{array}{l}\text { Lower } \\
\text { Bound }\end{array}$ & $\begin{array}{l}\text { Upper } \\
\text { Bound }\end{array}$ & $\begin{array}{l}\text { Lower } \\
\text { Bound }\end{array}$ & $\begin{array}{l}\text { Upper } \\
\text { Bound }\end{array}$ \\
\hline Forest Ecosystem C Pools & $(46.1)$ & $(52.8)$ & $(39.4)$ & $-14.5 \%$ & $14.5 \%$ \\
\hline
\end{tabular}

Table 2: Quantitative Uncertainty Estimates of Non- $\mathrm{CO}_{2}$ Emissions from Forest Fires $\left(\mathrm{MMT}^{\mathrm{CO}} \mathrm{O}_{2}\right.$ Eq. and Percent), Alabama $^{\mathrm{a}}$

\begin{tabular}{|c|c|c|c|c|c|c|}
\hline \multirow[b]{2}{*}{ Source } & \multirow[b]{2}{*}{ Gas } & \multirow[b]{2}{*}{$\begin{array}{l}2019 \text { Emission Estimate } \\
\text { (MMT CO2 Eq.) }\end{array}$} & \multicolumn{4}{|c|}{$\begin{array}{l}\text { Uncertainty Range Relative to Emission Estimate }{ }^{\mathrm{b}} \\
\left(\mathrm{MMT} \mathrm{CO}_{2} \text { Eq.) }\right.\end{array}$} \\
\hline & & & $\begin{array}{l}\text { Lower } \\
\text { Bound }\end{array}$ & $\begin{array}{l}\text { Upper } \\
\text { Bound }\end{array}$ & $\begin{array}{l}\text { Lower } \\
\text { Bound }\end{array}$ & $\begin{array}{l}\text { Upper } \\
\text { Bound }\end{array}$ \\
\hline Non- $\mathrm{CO}_{2}$ Emissions from Forest Fires & $\mathrm{CH}_{4}$ & + & + & 0.1 & $-28.8 \%$ & $31.6 \%$ \\
\hline Non- $\mathrm{CO}_{2}$ Emissions from Forest Fires & $\mathrm{N}_{2} \mathrm{O}$ & + & + & + & $-25.6 \%$ & $27.6 \%$ \\
\hline
\end{tabular}

+ Absolute value does not exceed $0.05 \mathrm{MMT} \mathrm{CO}_{2} \mathrm{Eq}$.

a These estimates include non- $\mathrm{CO}_{2}$ emissions from forest fires on Forest Land Remaining Forest Land and Land Converted to Forest Land.

${ }^{\mathrm{b}}$ Range of flux estimates predicted by Monte Carlo Stochastic Simulation for a 95 percent confidence interval, IPCC Approach 2. 


\section{Alaska}

Table 3: Quantitative Uncertainty Estimates for Net $\mathrm{CO}_{2}$ Flux from Forest Land Remaining Forest Land (MMT $\mathrm{CO}_{2}$ Eq. and Percent), Alaska

\begin{tabular}{|c|c|c|c|c|c|}
\hline \multirow[b]{3}{*}{ Source } & \multirow[b]{3}{*}{$\begin{array}{l}2019 \text { Flux Estimate } \\
\text { (MMT } \mathrm{CO}_{2} \text { Eq.) }\end{array}$} & \multicolumn{4}{|c|}{ Uncertainty Range Relative to Emission Estimate } \\
\hline & & \multicolumn{2}{|c|}{ (MMT CO 2 Eq.) } & \multicolumn{2}{|c|}{$(\%)$} \\
\hline & & $\begin{array}{l}\text { Lower } \\
\text { Bound }\end{array}$ & $\begin{array}{l}\text { Upper } \\
\text { Bound }\end{array}$ & $\begin{array}{l}\text { Lower } \\
\text { Bound }\end{array}$ & $\begin{array}{l}\text { Upper } \\
\text { Bound }\end{array}$ \\
\hline Forest Ecosystem C Pools & $(16.7)$ & $(21.9)$ & $(11.5)$ & $-31.2 \%$ & $31.2 \%$ \\
\hline
\end{tabular}

${ }^{a}$ Range of flux estimates predicted through a combination of sample-based and model-based uncertainty for a 95 percent confidence interval, IPCC Approach 1.

Notes: Parentheses indicate negative values or net uptake. Totals may not sum due to independent rounding.

Table 4: Quantitative Uncertainty Estimates of Non- $\mathrm{CO}_{2}$ Emissions from Forest Fires $\left(\mathrm{MMT}_{\mathrm{CO}}\right.$ Eq. and Percent), Alaska $^{\mathrm{a}}$

\begin{tabular}{|c|c|c|c|c|c|c|}
\hline \multirow[b]{3}{*}{ Source } & \multirow[b]{3}{*}{ Gas } & \multirow[b]{3}{*}{$\begin{array}{l}2019 \text { Emission Estimate } \\
\text { (MMT CO2 Eq.) }\end{array}$} & \multicolumn{4}{|c|}{ Uncertainty Range Relative to Emission Estimate ${ }^{\mathrm{b}}$} \\
\hline & & & \multicolumn{2}{|c|}{$\left(\mathrm{MMT} \mathrm{CO}_{2}\right.$ Eq.) } & \multicolumn{2}{|c|}{$(\%)$} \\
\hline & & & $\begin{array}{l}\text { Lower } \\
\text { Bound }\end{array}$ & $\begin{array}{l}\text { Upper } \\
\text { Bound }\end{array}$ & $\begin{array}{l}\text { Lower } \\
\text { Bound }\end{array}$ & $\begin{array}{l}\text { Upper } \\
\text { Bound }\end{array}$ \\
\hline Non- $\mathrm{CO}_{2}$ Emissions from Forest Fires & $\mathrm{CH}_{4}$ & 0.2 & 0.1 & 0.3 & $-42.8 \%$ & $51.1 \%$ \\
\hline Non- $\mathrm{CO}_{2}$ Emissions from Forest Fires & $\mathrm{N}_{2} \mathrm{O}$ & 0.1 & 0.1 & 0.2 & $-39.5 \%$ & $45.8 \%$ \\
\hline
\end{tabular}

a These estimates include non- $\mathrm{CO}_{2}$ emissions from forest fires on Forest Land Remaining Forest Land and Land Converted to Forest Land.

${ }^{\mathrm{b}}$ Range of flux estimates predicted by Monte Carlo Stochastic Simulation for a 95 percent confidence interval, IPCC Approach 2. 


\section{Arizona}

Table 5: Quantitative Uncertainty Estimates for Net $\mathrm{CO}_{2}$ Flux from Forest Land Remaining Forest Land (MMT $\mathrm{CO}_{2}$ Eq. and Percent), Arizona

\begin{tabular}{|c|c|c|c|c|c|}
\hline \multirow[b]{2}{*}{ Source } & \multirow[b]{2}{*}{$\begin{array}{l}2019 \text { Flux Estimate } \\
\left(\mathrm{MMT} \mathrm{CO}_{2} \text { Eq.) }\right.\end{array}$} & $\begin{array}{r}\text { Uncert: } \\
(\mathrm{MM}\end{array}$ & $\begin{array}{l}\text { (ange Re } \\
\text { Eq.) }\end{array}$ & to Emiss & imate $^{a}$ \\
\hline & & $\begin{array}{l}\text { Lower } \\
\text { Bound }\end{array}$ & $\begin{array}{l}\text { Upper } \\
\text { Bound }\end{array}$ & $\begin{array}{l}\text { Lower } \\
\text { Bound }\end{array}$ & $\begin{array}{l}\text { Upper } \\
\text { Bound }\end{array}$ \\
\hline Forest Ecosystem C Pools & 2.2 & 0.8 & 3.7 & $-64.7 \%$ & $64.7 \%$ \\
\hline
\end{tabular}

${ }^{a}$ Range of flux estimates predicted through a combination of sample-based and model-based uncertainty for a 95 percent confidence interval, IPCC Approach 1.

Notes: Parentheses indicate negative values or net uptake. Totals may not sum due to independent rounding.

Table 6: Quantitative Uncertainty Estimates of Non- $\mathrm{CO}_{2}$ Emissions from Forest Fires $\left(\mathrm{MMT}^{\mathrm{CO}} \mathrm{O}_{2}\right.$ Eq. and Percent), Arizona $^{\mathrm{a}}$

\begin{tabular}{|c|c|c|c|c|c|c|}
\hline \multirow[b]{2}{*}{ Source } & \multirow[b]{2}{*}{ Gas } & \multirow[b]{2}{*}{$\begin{array}{l}2019 \text { Emission Estimate } \\
\text { (MMT CO2 Eq.) }\end{array}$} & \multicolumn{4}{|c|}{$\begin{array}{l}\text { Uncertainty Range Relative to Emission Estimate } \\
\text { (MMT } \mathrm{CO}_{2} \text { Eq.) }\end{array}$} \\
\hline & & & $\begin{array}{l}\text { Lower } \\
\text { Bound }\end{array}$ & $\begin{array}{l}\text { Upper } \\
\text { Bound }\end{array}$ & $\begin{array}{l}\text { Lower } \\
\text { Bound }\end{array}$ & $\begin{array}{l}\text { Upper } \\
\text { Bound }\end{array}$ \\
\hline Non- $\mathrm{CO}_{2}$ Emissions from Forest Fires & $\mathrm{CH}_{4}$ & 0.1 & 0.1 & 0.2 & $-33 \%$ & $39.8 \%$ \\
\hline Non- $\mathrm{CO}_{2}$ Emissions from Forest Fires & $\mathrm{N}_{2} \mathrm{O}$ & 0.1 & 0.1 & 0.1 & $-27.7 \%$ & $32.4 \%$ \\
\hline
\end{tabular}

a These estimates include non- $\mathrm{CO}_{2}$ emissions from forest fires on Forest Land Remaining Forest Land and Land Converted to Forest Land.

${ }^{\mathrm{b}}$ Range of flux estimates predicted by Monte Carlo Stochastic Simulation for a 95 percent confidence interval, IPCC Approach 2. 


\section{Arkansas}

Table 7: Quantitative Uncertainty Estimates for Net $\mathrm{CO}_{2}$ Flux from Forest Land Remaining Forest Land (MMT $\mathrm{CO}_{2}$ Eq. and Percent), Arkansas

\begin{tabular}{|c|c|c|c|c|c|}
\hline \multirow[b]{3}{*}{ Source } & \multirow{3}{*}{$\begin{array}{l}2019 \text { Flux Estimate } \\
\text { (MMT } \mathrm{CO}_{2} \text { Eq.) }\end{array}$} & \multicolumn{4}{|c|}{ Uncertainty Range Relative to Emission Estimate $^{a}$} \\
\hline & & \multicolumn{2}{|c|}{ (MMT $\mathrm{CO}_{2}$ Eq.) } & \multicolumn{2}{|c|}{$(\%)$} \\
\hline & & Lower & Upper & Lower & Upper \\
\hline Forest Ecosystem C Pools & $(29.9)$ & $(35.7)$ & $(24.2)$ & $-19.2 \%$ & $19.2 \%$ \\
\hline
\end{tabular}

${ }^{a}$ Range of flux estimates predicted through a combination of sample-based and model-based uncertainty for a 95 percent confidence interval, IPCC Approach 1.

Notes: Parentheses indicate negative values or net uptake. Totals may not sum due to independent rounding.

Table 8: Quantitative Uncertainty Estimates of Non- $\mathrm{CO}_{2}$ Emissions from Forest Fires $\left(\mathrm{MMT}^{\mathrm{CO}} \mathrm{O}_{2}\right.$ Eq. and Percent), Arkansas $^{\mathrm{a}}$

\begin{tabular}{|c|c|c|c|c|c|c|}
\hline \multirow[b]{2}{*}{ Source } & \multirow[b]{2}{*}{ Gas } & \multirow[b]{2}{*}{$\begin{array}{l}2019 \text { Emission Estimate } \\
\text { (MMT CO2 Eq.) }\end{array}$} & \multicolumn{4}{|c|}{$\begin{array}{l}\text { Uncertainty Range Relative to Emission Estimate }{ }^{\mathrm{b}} \\
\left(\mathrm{MMT} \mathrm{CO}_{2} \text { Eq.) }\right.\end{array}$} \\
\hline & & & $\begin{array}{l}\text { Lower } \\
\text { Bound }\end{array}$ & $\begin{array}{l}\text { Upper } \\
\text { Bound }\end{array}$ & $\begin{array}{l}\text { Lower } \\
\text { Bound }\end{array}$ & $\begin{array}{l}\text { Upper } \\
\text { Bound }\end{array}$ \\
\hline Non- $\mathrm{CO}_{2}$ Emissions from Forest Fires & $\mathrm{CH}_{4}$ & 0.1 & 0.1 & 0.1 & $-27.6 \%$ & $32.6 \%$ \\
\hline Non- $\mathrm{CO}_{2}$ Emissions from Forest Fires & $\mathrm{N}_{2} \mathrm{O}$ & 0.1 & + & 0.1 & $-24.8 \%$ & $28.3 \%$ \\
\hline
\end{tabular}

+ Absolute value does not exceed 0.05 MMT $\mathrm{CO}_{2} \mathrm{Eq}$.

a These estimates include non- $\mathrm{CO}_{2}$ emissions from forest fires on Forest Land Remaining Forest Land and Land Converted to Forest Land.

${ }^{\mathrm{b}}$ Range of flux estimates predicted by Monte Carlo Stochastic Simulation for a 95 percent confidence interval, IPCC Approach 2. 


\section{California}

Table 9: Quantitative Uncertainty Estimates for Net $\mathrm{CO}_{2}$ Flux from Forest Land Remaining Forest Land (MMT $\mathrm{CO}_{2}$ Eq. and Percent), California

\begin{tabular}{|c|c|c|c|c|c|}
\hline \multirow[b]{3}{*}{ Source } & \multirow[b]{3}{*}{$\begin{array}{l}2019 \text { Flux Estimate } \\
\text { (MMT } \mathrm{CO}_{2} \text { Eq.) }\end{array}$} & \multicolumn{4}{|c|}{ Uncertainty Range Relative to Emission Estimate ${ }^{a}$} \\
\hline & & \multicolumn{2}{|c|}{ (MMT CO $\mathrm{Cq}_{2}$ Eq) } & \multicolumn{2}{|c|}{$(\%)$} \\
\hline & & Lower & Upper & Lower & Upper \\
\hline Forest Ecosystem C Pools & $(26.8)$ & $(56.1)$ & 2.5 & $-109.5 \%$ & $109.5 \%$ \\
\hline
\end{tabular}

${ }^{a}$ Range of flux estimates predicted through a combination of sample-based and model-based uncertainty for a 95 percent confidence interval, IPCC Approach 1.

Notes: Parentheses indicate negative values or net uptake. Totals may not sum due to independent rounding.

Table 10: Quantitative Uncertainty Estimates of Non- $\mathrm{CO}_{2}$ Emissions from Forest Fires (MMT $\mathrm{CO}_{2}$ Eq. and Percent), California $^{a}$

\begin{tabular}{|c|c|c|c|c|c|c|}
\hline \multirow[b]{2}{*}{ Source } & \multirow[b]{2}{*}{ Gas } & \multirow[b]{2}{*}{$\begin{array}{l}2019 \text { Emission Estimate } \\
\text { (MMT CO2 Eq.) }\end{array}$} & \multicolumn{4}{|c|}{$\begin{array}{l}\text { Uncertainty Range Relative to Emission Estimate } \\
\text { (MMT } \mathrm{CO}_{2} \text { Eq.) }\end{array}$} \\
\hline & & & $\begin{array}{l}\text { Lower } \\
\text { Bound }\end{array}$ & $\begin{array}{l}\text { Upper } \\
\text { Bound }\end{array}$ & $\begin{array}{l}\text { Lower } \\
\text { Bound }\end{array}$ & $\begin{array}{l}\text { Upper } \\
\text { Bound }\end{array}$ \\
\hline Non- $\mathrm{CO}_{2}$ Emissions from Forest Fires & $\mathrm{CH}_{4}$ & 4.2 & 2.8 & 5.7 & $-31.6 \%$ & $36.5 \%$ \\
\hline Non- $\mathrm{CO}_{2}$ Emissions from Forest Fires & $\mathrm{N}_{2} \mathrm{O}$ & 2.7 & 2.1 & 3.5 & $-25 \%$ & $28.1 \%$ \\
\hline
\end{tabular}

a These estimates include non- $\mathrm{CO}_{2}$ emissions from forest fires on Forest Land Remaining Forest Land and Land Converted to Forest Land.

${ }^{\mathrm{b}}$ Range of flux estimates predicted by Monte Carlo Stochastic Simulation for a 95 percent confidence interval, IPCC Approach 2. 


\section{Colorado}

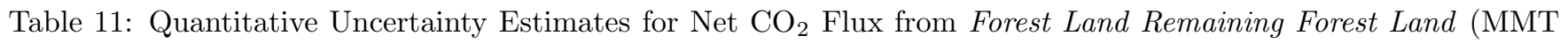
$\mathrm{CO}_{2}$ Eq. and Percent), Colorado

\begin{tabular}{|c|c|c|c|c|c|}
\hline \multirow[b]{3}{*}{ Source } & \multirow[b]{3}{*}{$\begin{array}{l}2019 \text { Flux Estimate } \\
\text { (MMT } \mathrm{CO}_{2} \text { Eq.) }\end{array}$} & \multicolumn{4}{|c|}{ Uncertainty Range Relative to Emission Estimate ${ }^{a}$} \\
\hline & & \multicolumn{2}{|c|}{ (MMT $\mathrm{CO}_{2}$ Eq.) } & \multicolumn{2}{|c|}{$(\%)$} \\
\hline & & $\begin{array}{l}\text { Lower } \\
\text { Bound }\end{array}$ & $\begin{array}{l}\text { Upper } \\
\text { Bound }\end{array}$ & $\begin{array}{l}\text { Lower } \\
\text { Bound }\end{array}$ & $\begin{array}{l}\text { Upper } \\
\text { Bound }\end{array}$ \\
\hline Forest Ecosystem C Pools & 11.7 & $(19.6)$ & 42.9 & $-267.6 \%$ & $267.6 \%$ \\
\hline
\end{tabular}

${ }^{a}$ Range of flux estimates predicted through a combination of sample-based and model-based uncertainty for a 95 percent confidence interval, IPCC Approach 1.

Notes: Parentheses indicate negative values or net uptake. Totals may not sum due to independent rounding.

Table 12: Quantitative Uncertainty Estimates of Non- $\mathrm{CO}_{2}$ Emissions from Forest Fires $\left(\mathrm{MMT} \mathrm{CO}_{2}\right.$ Eq. and Percent), Colorado ${ }^{\mathrm{a}}$

\begin{tabular}{|c|c|c|c|c|c|c|}
\hline \multirow[b]{3}{*}{ Source } & \multirow[b]{3}{*}{ Gas } & \multirow[b]{3}{*}{$\begin{array}{l}2019 \text { Emission Estimate } \\
\text { (MMT CO2 Eq.) }\end{array}$} & \multicolumn{4}{|c|}{ Uncertainty Range Relative to Emission Estimate ${ }^{b}$} \\
\hline & & & \multicolumn{2}{|c|}{$(\mathrm{MMT} \mathrm{CO} 2$ Eq.) } & \multicolumn{2}{|c|}{$(\%)$} \\
\hline & & & $\begin{array}{l}\text { Lower } \\
\text { Bound }\end{array}$ & $\begin{array}{l}\text { Upper } \\
\text { Bound }\end{array}$ & $\begin{array}{l}\text { Lower } \\
\text { Bound }\end{array}$ & $\begin{array}{l}\text { Upper } \\
\text { Bound }\end{array}$ \\
\hline Non- $\mathrm{CO}_{2}$ Emissions from Forest Fires & $\mathrm{CH}_{4}$ & 0.6 & 0.3 & 0.8 & $-37.5 \%$ & $44.9 \%$ \\
\hline Non- $\mathrm{CO}_{2}$ Emissions from Forest Fires & $\mathrm{N}_{2} \mathrm{O}$ & 0.4 & 0.3 & 0.5 & $-29.6 \%$ & $36.1 \%$ \\
\hline
\end{tabular}

a These estimates include non- $\mathrm{CO}_{2}$ emissions from forest fires on Forest Land Remaining Forest Land and Land Converted to Forest Land.

${ }^{\mathrm{b}}$ Range of flux estimates predicted by Monte Carlo Stochastic Simulation for a 95 percent confidence interval, IPCC Approach 2. 


\section{Connecticut}

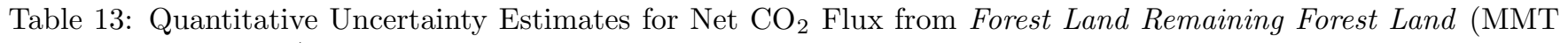
$\mathrm{CO}_{2}$ Eq. and Percent), Connecticut

\begin{tabular}{|c|c|c|c|c|c|}
\hline \multirow[b]{3}{*}{ Source } & \multirow{3}{*}{$\begin{array}{l}2019 \text { Flux Estimate } \\
\text { (MMT } \mathrm{CO}_{2} \text { Eq.) }\end{array}$} & \multicolumn{4}{|c|}{ Uncertainty Range Relative to Emission Estimate } \\
\hline & & \multicolumn{2}{|c|}{ (MMT CO 2 Eq.) } & \multicolumn{2}{|c|}{$(\%)$} \\
\hline & & Lower & Upper & Lower & Upper \\
\hline & & & & & \\
\hline Forest Ecosystem C Pools & $(3.2)$ & $(4.2)$ & $(2.2)$ & $-32.4 \%$ & $32.4 \%$ \\
\hline
\end{tabular}

${ }^{a}$ Range of flux estimates predicted through a combination of sample-based and model-based uncertainty for a 95 percent confidence interval, IPCC Approach 1.

Notes: Parentheses indicate negative values or net uptake. Totals may not sum due to independent rounding. 


\section{Delaware}

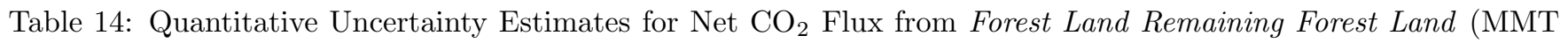
$\mathrm{CO}_{2}$ Eq. and Percent), Delaware

\begin{tabular}{|c|c|c|c|c|c|}
\hline \multirow[b]{3}{*}{ Source } & \multirow{3}{*}{$\begin{array}{l}2019 \text { Flux Estimate } \\
\text { (MMT } \mathrm{CO}_{2} \text { Eq.) }\end{array}$} & \multicolumn{4}{|c|}{ Uncertainty Range Relative to Emission Estimate } \\
\hline & & \multicolumn{2}{|c|}{ (MMT CO 2 Eq.) } & \multicolumn{2}{|c|}{$(\%)$} \\
\hline & & Lower & Upper & Lower & Upper \\
\hline & & & & & \\
\hline Forest Ecosystem C Pools & $(0.2)$ & $(0.4)$ & $(0.1)$ & $-47.4 \%$ & $47.4 \%$ \\
\hline
\end{tabular}

${ }^{a}$ Range of flux estimates predicted through a combination of sample-based and model-based uncertainty for a 95 percent confidence interval, IPCC Approach 1.

Notes: Parentheses indicate negative values or net uptake. Totals may not sum due to independent rounding. 


\section{Florida}

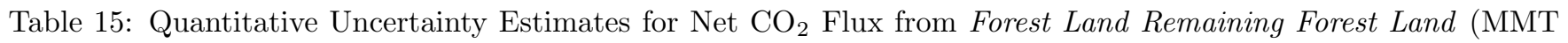
$\mathrm{CO}_{2}$ Eq. and Percent), Florida

\begin{tabular}{|c|c|c|c|c|c|}
\hline \multirow[b]{3}{*}{ Source } & \multirow[b]{3}{*}{$\begin{array}{l}2019 \text { Flux Estimate } \\
\text { (MMT } \mathrm{CO}_{2} \text { Eq.) }\end{array}$} & \multicolumn{4}{|c|}{ Uncertainty Range Relative to Emission Estimate } \\
\hline & & \multicolumn{2}{|c|}{ (MMT CO 2 Eq.) } & \multicolumn{2}{|c|}{$(\%)$} \\
\hline & & $\begin{array}{l}\text { Lower } \\
\text { Bound }\end{array}$ & $\begin{array}{l}\text { Upper } \\
\text { Bound }\end{array}$ & $\begin{array}{l}\text { Lower } \\
\text { Bound }\end{array}$ & $\begin{array}{l}\text { Upper } \\
\text { Bound }\end{array}$ \\
\hline Forest Ecosystem C Pools & $(19.7)$ & $(22.1)$ & $(17.2)$ & $-12.4 \%$ & $12.4 \%$ \\
\hline
\end{tabular}

${ }^{a}$ Range of flux estimates predicted through a combination of sample-based and model-based uncertainty for a 95 percent confidence interval, IPCC Approach 1.

Notes: Parentheses indicate negative values or net uptake. Totals may not sum due to independent rounding.

Table 16: Quantitative Uncertainty Estimates of Non- $\mathrm{CO}_{2}$ Emissions from Forest Fires $\left(\mathrm{MMT} \mathrm{CO}_{2}\right.$ Eq. and Percent), Florida $^{a}$

\begin{tabular}{|c|c|c|c|c|c|c|}
\hline \multirow[b]{3}{*}{ Source } & \multirow[b]{3}{*}{ Gas } & \multirow[b]{3}{*}{$\begin{array}{l}2019 \text { Emission Estimate } \\
\text { (MMT CO2 Eq.) }\end{array}$} & \multicolumn{4}{|c|}{ Uncertainty Range Relative to Emission Estimate ${ }^{\mathrm{b}}$} \\
\hline & & & \multicolumn{2}{|c|}{$\left(\mathrm{MMT} \mathrm{CO}_{2}\right.$ Eq.) } & \multicolumn{2}{|c|}{$(\%)$} \\
\hline & & & $\begin{array}{l}\text { Lower } \\
\text { Bound }\end{array}$ & $\begin{array}{l}\text { Upper } \\
\text { Bound }\end{array}$ & $\begin{array}{l}\text { Lower } \\
\text { Bound }\end{array}$ & $\begin{array}{l}\text { Upper } \\
\text { Bound }\end{array}$ \\
\hline Non- $\mathrm{CO}_{2}$ Emissions from Forest Fires & $\mathrm{CH}_{4}$ & 0.2 & 0.1 & 0.2 & $-18.3 \%$ & $20.6 \%$ \\
\hline Non- $\mathrm{CO}_{2}$ Emissions from Forest Fires & $\mathrm{N}_{2} \mathrm{O}$ & 0.1 & 0.1 & 0.1 & $-16.1 \%$ & $18.1 \%$ \\
\hline
\end{tabular}

a These estimates include non- $\mathrm{CO}_{2}$ emissions from forest fires on Forest Land Remaining Forest Land and Land Converted to Forest Land.

${ }^{\mathrm{b}}$ Range of flux estimates predicted by Monte Carlo Stochastic Simulation for a 95 percent confidence interval, IPCC Approach 2. 


\section{Georgia}

Table 17: Quantitative Uncertainty Estimates for Net $\mathrm{CO}_{2}$ Flux from Forest Land Remaining Forest Land (MMT $\mathrm{CO}_{2}$ Eq. and Percent), Georgia

\begin{tabular}{|c|c|c|c|c|c|}
\hline \multirow[b]{3}{*}{ Source } & \multirow[b]{3}{*}{$\begin{array}{l}2019 \text { Flux Estimate } \\
\text { (MMT } \mathrm{CO}_{2} \text { Eq.) }\end{array}$} & \multicolumn{4}{|c|}{ Uncertainty Range Relative to Emission Estimate } \\
\hline & & \multicolumn{2}{|c|}{ (MMT $\mathrm{CO}_{2}$ Eq.) } & \multicolumn{2}{|c|}{$(\%)$} \\
\hline & & Lower & Upper & Lower & Upper \\
\hline Forest Ecosystem C Pools & $(30.2)$ & $(32.0)$ & $(28.4)$ & $-6 \%$ & $6 \%$ \\
\hline
\end{tabular}

Table 18: Quantitative Uncertainty Estimates of Non- $\mathrm{CO}_{2}$ Emissions from Forest Fires $\left(\mathrm{MMT} \mathrm{CO}_{2}\right.$ Eq. and Percent), Georgia $^{a}$

\begin{tabular}{|c|c|c|c|c|c|c|}
\hline \multirow[b]{3}{*}{ Source } & \multirow[b]{3}{*}{ Gas } & \multirow[b]{3}{*}{$\begin{array}{l}2019 \text { Emission Estimate } \\
\text { (MMT CO2 Eq.) }\end{array}$} & \multicolumn{4}{|c|}{ Uncertainty Range Relative to Emission Estimate ${ }^{\mathrm{b}}$} \\
\hline & & & \multicolumn{2}{|c|}{ (MMT $\mathrm{CO}_{2}$ Eq.) } & \multicolumn{2}{|c|}{$(\%)$} \\
\hline & & & $\begin{array}{l}\text { Lower } \\
\text { Bound }\end{array}$ & $\begin{array}{l}\text { Upper } \\
\text { Bound }\end{array}$ & $\begin{array}{l}\text { Lower } \\
\text { Bound }\end{array}$ & $\begin{array}{l}\text { Upper } \\
\text { Bound }\end{array}$ \\
\hline Non- $\mathrm{CO}_{2}$ Emissions from Forest Fires & $\mathrm{CH}_{4}$ & + & + & 0.1 & $-71.4 \%$ & $122.8 \%$ \\
\hline Non- $\mathrm{CO}_{2}$ Emissions from Forest Fires & $\mathrm{N}_{2} \mathrm{O}$ & + & + & + & $-67.6 \%$ & $104.7 \%$ \\
\hline
\end{tabular}

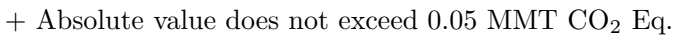

a These estimates include non- $\mathrm{CO}_{2}$ emissions from forest fires on Forest Land Remaining Forest Land and Land Converted to Forest Land.

${ }^{\mathrm{b}}$ Range of flux estimates predicted by Monte Carlo Stochastic Simulation for a 95 percent confidence interval, IPCC Approach 2. 


\section{Idaho}

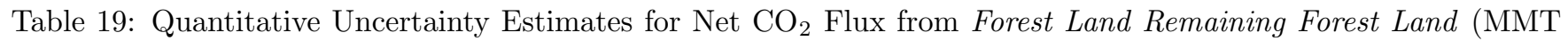
$\mathrm{CO}_{2}$ Eq. and Percent), Idaho

\begin{tabular}{|c|c|c|c|c|c|}
\hline \multirow[b]{3}{*}{ Source } & \multirow[b]{3}{*}{$\begin{array}{l}2019 \text { Flux Estimate } \\
\text { (MMT } \mathrm{CO}_{2} \text { Eq.) }\end{array}$} & \multicolumn{4}{|c|}{ Uncertainty Range Relative to Emission Estimate } \\
\hline & & \multicolumn{2}{|c|}{ (MMT CO 2 Eq.) } & \multicolumn{2}{|c|}{$(\%)$} \\
\hline & & $\begin{array}{l}\text { Lower } \\
\text { Bound }\end{array}$ & $\begin{array}{l}\text { Upper } \\
\text { Bound }\end{array}$ & $\begin{array}{l}\text { Lower } \\
\text { Bound }\end{array}$ & $\begin{array}{l}\text { Upper } \\
\text { Bound }\end{array}$ \\
\hline Forest Ecosystem C Pools & 4.6 & (8.3) & 17.5 & $-278.9 \%$ & $278.9 \%$ \\
\hline
\end{tabular}

${ }^{a}$ Range of flux estimates predicted through a combination of sample-based and model-based uncertainty for a 95 percent confidence interval, IPCC Approach 1.

Notes: Parentheses indicate negative values or net uptake. Totals may not sum due to independent rounding.

Table 20: Quantitative Uncertainty Estimates of Non- $\mathrm{CO}_{2}$ Emissions from Forest Fires $\left(\mathrm{MMT} \mathrm{CO}_{2}\right.$ Eq. and Percent), Idaho $^{\mathrm{a}}$

\begin{tabular}{|c|c|c|c|c|c|c|}
\hline \multirow[b]{2}{*}{ Source } & \multirow[b]{2}{*}{ Gas } & \multirow[b]{2}{*}{$\begin{array}{l}2019 \text { Emission Estimate } \\
\text { (MMT CO2 Eq.) }\end{array}$} & \multicolumn{4}{|c|}{$\begin{array}{l}\text { Uncertainty Range Relative to Emission Estimate } \\
\text { (MMT } \mathrm{CO}_{2} \text { Eq.) }\end{array}$} \\
\hline & & & $\begin{array}{l}\text { Lower } \\
\text { Bound }\end{array}$ & $\begin{array}{l}\text { Upper } \\
\text { Bound }\end{array}$ & $\begin{array}{l}\text { Lower } \\
\text { Bound }\end{array}$ & $\begin{array}{l}\text { Upper } \\
\text { Bound }\end{array}$ \\
\hline Non- $\mathrm{CO}_{2}$ Emissions from Forest Fires & $\mathrm{CH}_{4}$ & 0.3 & 0.2 & 0.5 & $-29.6 \%$ & $33.9 \%$ \\
\hline Non- $\mathrm{CO}_{2}$ Emissions from Forest Fires & $\mathrm{N}_{2} \mathrm{O}$ & 0.2 & 0.2 & 0.3 & $-22.6 \%$ & $26.2 \%$ \\
\hline
\end{tabular}

a These estimates include non- $\mathrm{CO}_{2}$ emissions from forest fires on Forest Land Remaining Forest Land and Land Converted to Forest Land.

${ }^{\mathrm{b}}$ Range of flux estimates predicted by Monte Carlo Stochastic Simulation for a 95 percent confidence interval, IPCC Approach 2. 


\section{Illinois}

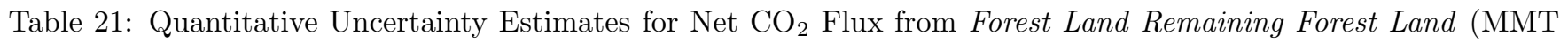
$\mathrm{CO}_{2}$ Eq. and Percent), Illinois

\begin{tabular}{|c|c|c|c|c|c|}
\hline \multirow[b]{3}{*}{ Source } & \multirow{3}{*}{$\begin{array}{l}2019 \text { Flux Estimate } \\
\text { (MMT } \mathrm{CO}_{2} \text { Eq.) }\end{array}$} & \multicolumn{4}{|c|}{ Uncertainty Range Relative to Emission Estimate $^{a}$} \\
\hline & & \multicolumn{2}{|c|}{ (MMT $\mathrm{CO}_{2}$ Eq.) } & \multicolumn{2}{|c|}{$(\%)$} \\
\hline & & Lower & Upper & Lower & Upper \\
\hline Forest Ecosystem C Pools & $(4.4)$ & $(8.0)$ & $(0.8)$ & $-82.5 \%$ & $82.5 \%$ \\
\hline
\end{tabular}

${ }^{a}$ Range of flux estimates predicted through a combination of sample-based and model-based uncertainty for a 95 percent confidence interval, IPCC Approach 1.

Notes: Parentheses indicate negative values or net uptake. Totals may not sum due to independent rounding.

Table 22: Quantitative Uncertainty Estimates of Non- $\mathrm{CO}_{2}$ Emissions from Forest Fires $\left(\mathrm{MMT} \mathrm{CO}_{2}\right.$ Eq. and Percent), Illinois $^{\mathrm{a}}$

\begin{tabular}{|c|c|c|c|c|c|c|}
\hline \multirow[b]{2}{*}{ Source } & \multirow[b]{2}{*}{ Gas } & \multirow[b]{2}{*}{$\begin{array}{l}2019 \text { Emission Estimate } \\
\text { (MMT CO2 Eq.) }\end{array}$} & \multicolumn{4}{|c|}{$\begin{array}{l}\text { Uncertainty Range Relative to Emission Estimate } \\
\text { (MMT } \mathrm{CO}_{2} \text { Eq.) }\end{array}$} \\
\hline & & & $\begin{array}{l}\text { Lower } \\
\text { Bound }\end{array}$ & $\begin{array}{l}\text { Upper } \\
\text { Bound }\end{array}$ & $\begin{array}{l}\text { Lower } \\
\text { Bound }\end{array}$ & $\begin{array}{l}\text { Upper } \\
\text { Bound }\end{array}$ \\
\hline Non- $\mathrm{CO}_{2}$ Emissions from Forest Fires & $\mathrm{CH}_{4}$ & + & + & + & $-76.3 \%$ & $105.2 \%$ \\
\hline Non- $\mathrm{CO}_{2}$ Emissions from Forest Fires & $\mathrm{N}_{2} \mathrm{O}$ & + & + & + & $-72.9 \%$ & $91.5 \%$ \\
\hline
\end{tabular}

+ Absolute value does not exceed 0.05 MMT $\mathrm{CO}_{2}$ Eq.

${ }^{a}$ These estimates include non- $\mathrm{CO}_{2}$ emissions from forest fires on Forest Land Remaining Forest Land and Land Converted to Forest Land.

${ }^{\mathrm{b}}$ Range of flux estimates predicted by Monte Carlo Stochastic Simulation for a 95 percent confidence interval, IPCC Approach 2. 


\section{Indiana}

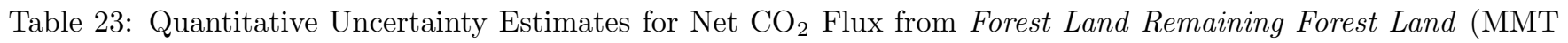
$\mathrm{CO}_{2}$ Eq. and Percent), Indiana

\begin{tabular}{|c|c|c|c|c|c|}
\hline \multirow[b]{2}{*}{ Source } & \multirow[b]{2}{*}{$\begin{array}{l}2019 \text { Flux Estimate } \\
\text { (MMT } \mathrm{CO}_{2} \text { Eq.) }\end{array}$} & $\begin{array}{r}\text { Uncert } \\
(\mathrm{MM}\end{array}$ & $\begin{array}{l}\text { (ange Re } \\
\text { Eq.) }\end{array}$ & to Emissi & timate $^{\mathrm{a}}$ \\
\hline & & $\begin{array}{l}\text { Lower } \\
\text { Bound }\end{array}$ & $\begin{array}{l}\text { Upper } \\
\text { Bound }\end{array}$ & $\begin{array}{l}\text { Lower } \\
\text { Bound }\end{array}$ & $\begin{array}{l}\text { Upper } \\
\text { Bound }\end{array}$ \\
\hline Forest Ecosystem C Pools & $(5.2)$ & $(11.0)$ & 0.6 & $-112.5 \%$ & $112.5 \%$ \\
\hline
\end{tabular}

Table 24: Quantitative Uncertainty Estimates of Non- $\mathrm{CO}_{2}$ Emissions from Forest Fires $\left(\mathrm{MMT} \mathrm{CO}_{2}\right.$ Eq. and Percent), Indiana $^{a}$

\begin{tabular}{|c|c|c|c|c|c|c|}
\hline \multirow[b]{2}{*}{ Source } & \multirow[b]{2}{*}{ Gas } & \multirow[b]{2}{*}{$\begin{array}{l}2019 \text { Emission Estimate } \\
\text { (MMT CO2 Eq.) }\end{array}$} & \multicolumn{4}{|c|}{$\begin{array}{l}\text { Uncertainty Range Relative to Emission Estimate }{ }^{\mathrm{b}} \\
\left(\mathrm{MMT} \mathrm{CO}_{2} \text { Eq.) }\right.\end{array}$} \\
\hline & & & $\begin{array}{l}\text { Lower } \\
\text { Bound }\end{array}$ & $\begin{array}{l}\text { Upper } \\
\text { Bound }\end{array}$ & $\begin{array}{l}\text { Lower } \\
\text { Bound }\end{array}$ & $\begin{array}{l}\text { Upper } \\
\text { Bound }\end{array}$ \\
\hline Non- $\mathrm{CO}_{2}$ Emissions from Forest Fires & $\mathrm{CH}_{4}$ & + & + & + & $-78.8 \%$ & $120.5 \%$ \\
\hline Non- $\mathrm{CO}_{2}$ Emissions from Forest Fires & $\mathrm{N}_{2} \mathrm{O}$ & + & + & + & $-75.5 \%$ & $100.2 \%$ \\
\hline
\end{tabular}

+ Absolute value does not exceed $0.05 \mathrm{MMT} \mathrm{CO}_{2} \mathrm{Eq}$.

a These estimates include non- $\mathrm{CO}_{2}$ emissions from forest fires on Forest Land Remaining Forest Land and Land Converted to Forest Land.

${ }^{\mathrm{b}}$ Range of flux estimates predicted by Monte Carlo Stochastic Simulation for a 95 percent confidence interval, IPCC Approach 2. 


\section{Iowa}

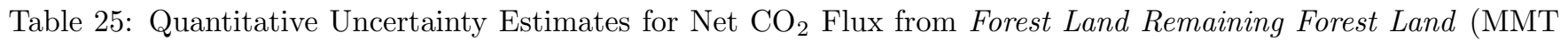
$\mathrm{CO}_{2}$ Eq. and Percent), Iowa

\begin{tabular}{|c|c|c|c|c|c|}
\hline \multirow[b]{3}{*}{ Source } & \multirow{3}{*}{$\begin{array}{l}2019 \text { Flux Estimate } \\
\text { (MMT } \mathrm{CO}_{2} \text { Eq.) }\end{array}$} & \multicolumn{4}{|c|}{ Uncertainty Range Relative to Emission Estimate } \\
\hline & & \multicolumn{2}{|c|}{ (MMT CO $\mathrm{Cq}_{2}$ Eq.) } & \multicolumn{2}{|c|}{$(\%)$} \\
\hline & & Lower & Upper & Lower & Upper \\
\hline Forest Ecosystem C Pools & $(2.6)$ & $(3.8)$ & $(1.5)$ & $-44.1 \%$ & $44.1 \%$ \\
\hline
\end{tabular}

${ }^{a}$ Range of flux estimates predicted through a combination of sample-based and model-based uncertainty for a 95 percent confidence interval, IPCC Approach 1.

Notes: Parentheses indicate negative values or net uptake. Totals may not sum due to independent rounding. 


\section{Kansas}

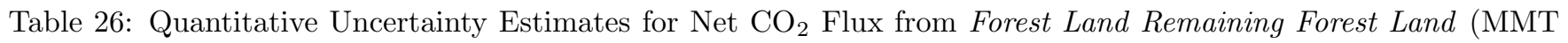
$\mathrm{CO}_{2}$ Eq. and Percent), Kansas

\begin{tabular}{|c|c|c|c|c|c|}
\hline \multirow[b]{3}{*}{ Source } & \multirow[b]{3}{*}{$\begin{array}{l}2019 \text { Flux Estimate } \\
\left(\mathrm{MMT} \mathrm{CO}_{2} \text { Eq.) }\right.\end{array}$} & \multicolumn{4}{|c|}{ Uncertainty Range Relative to Emission Estimate } \\
\hline & & \multicolumn{2}{|c|}{ (MMT $\mathrm{CO}_{2}$ Eq.) } & \multicolumn{2}{|c|}{$(\%)$} \\
\hline & & $\begin{array}{l}\text { Lower } \\
\text { Bound }\end{array}$ & $\begin{array}{l}\text { Upper } \\
\text { Bound }\end{array}$ & $\begin{array}{l}\text { Lower } \\
\text { Bound }\end{array}$ & $\begin{array}{l}\text { Upper } \\
\text { Bound }\end{array}$ \\
\hline Forest Ecosystem C Pools & $(2.3)$ & $(3.8)$ & $(0.7)$ & $-68.2 \%$ & $68.2 \%$ \\
\hline
\end{tabular}

Table 27: Quantitative Uncertainty Estimates of Non- $\mathrm{CO}_{2}$ Emissions from Forest Fires $\left(\mathrm{MMT} \mathrm{CO}_{2}\right.$ Eq. and Percent), Kansas $^{\mathrm{a}}$

\begin{tabular}{|c|c|c|c|c|c|c|}
\hline \multirow[b]{2}{*}{ Source } & \multirow[b]{2}{*}{ Gas } & \multirow[b]{2}{*}{$\begin{array}{l}2019 \text { Emission Estimate } \\
\text { (MMT CO2 Eq.) }\end{array}$} & \multicolumn{4}{|c|}{$\begin{array}{l}\text { Uncertainty Range Relative to Emission Estimate }{ }^{\mathrm{b}} \\
\left(\mathrm{MMT} \mathrm{CO}_{2} \text { Eq.) }\right.\end{array}$} \\
\hline & & & $\begin{array}{l}\text { Lower } \\
\text { Bound }\end{array}$ & $\begin{array}{l}\text { Upper } \\
\text { Bound }\end{array}$ & $\begin{array}{l}\text { Lower } \\
\text { Bound }\end{array}$ & $\begin{array}{l}\text { Upper } \\
\text { Bound }\end{array}$ \\
\hline Non- $\mathrm{CO}_{2}$ Emissions from Forest Fires & $\mathrm{CH}_{4}$ & + & + & + & $-86.5 \%$ & $152.5 \%$ \\
\hline Non- $\mathrm{CO}_{2}$ Emissions from Forest Fires & $\mathrm{N}_{2} \mathrm{O}$ & + & + & + & $-82.1 \%$ & $122.7 \%$ \\
\hline
\end{tabular}

+ Absolute value does not exceed 0.05 $\mathrm{MMT} \mathrm{CO}_{2} \mathrm{Eq}$.

a These estimates include non- $\mathrm{CO}_{2}$ emissions from forest fires on Forest Land Remaining Forest Land and Land Converted to Forest Land.

${ }^{\mathrm{b}}$ Range of flux estimates predicted by Monte Carlo Stochastic Simulation for a 95 percent confidence interval, IPCC Approach 2. 


\section{Kentucky}

Table 28: Quantitative Uncertainty Estimates for Net $\mathrm{CO}_{2}$ Flux from Forest Land Remaining Forest Land (MMT $\mathrm{CO}_{2}$ Eq. and Percent), Kentucky

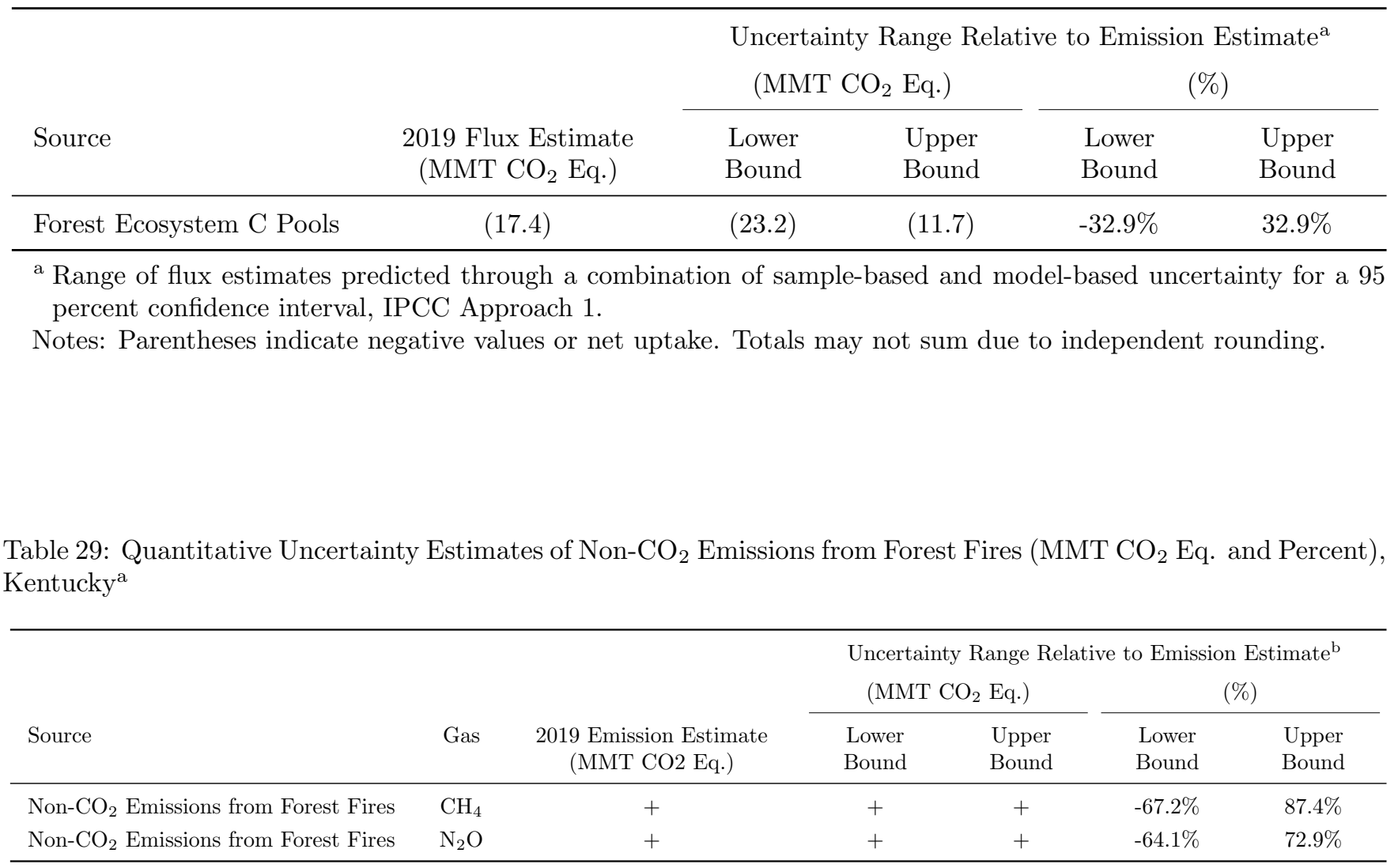

+ Absolute value does not exceed $0.05 \mathrm{MMT} \mathrm{CO}_{2} \mathrm{Eq}$.

a These estimates include non- $\mathrm{CO}_{2}$ emissions from forest fires on Forest Land Remaining Forest Land and Land Converted to Forest Land.

${ }^{\mathrm{b}}$ Range of flux estimates predicted by Monte Carlo Stochastic Simulation for a 95 percent confidence interval, IPCC Approach 2. 


\section{Louisiana}

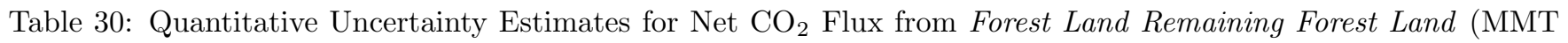
$\mathrm{CO}_{2}$ Eq. and Percent), Louisiana

\begin{tabular}{|c|c|c|c|c|c|}
\hline \multirow[b]{2}{*}{ Source } & \multirow[b]{2}{*}{$\begin{array}{l}2019 \text { Flux Estimate } \\
\left(\mathrm{MMT} \mathrm{CO}_{2} \text { Eq.) }\right.\end{array}$} & \multicolumn{3}{|c|}{ Uncertainty Range Relative to Emission Estimate ${ }^{a}$} & imate $^{\mathrm{a}}$ \\
\hline & & $\begin{array}{l}\text { Lower } \\
\text { Bound }\end{array}$ & $\begin{array}{l}\text { Upper } \\
\text { Bound }\end{array}$ & $\begin{array}{l}\text { Lower } \\
\text { Bound }\end{array}$ & $\begin{array}{l}\text { Upper } \\
\text { Bound }\end{array}$ \\
\hline Forest Ecosystem C Pools & $(22.2)$ & $(24.0)$ & $(20.5)$ & $-7.8 \%$ & $7.8 \%$ \\
\hline
\end{tabular}

${ }^{a}$ Range of flux estimates predicted through a combination of sample-based and model-based uncertainty for a 95 percent confidence interval, IPCC Approach 1.

Notes: Parentheses indicate negative values or net uptake. Totals may not sum due to independent rounding.

Table 31: Quantitative Uncertainty Estimates of Non- $\mathrm{CO}_{2}$ Emissions from Forest Fires $\left(\mathrm{MMT} \mathrm{CO}_{2}\right.$ Eq. and Percent), Louisiana $^{\mathrm{a}}$

\begin{tabular}{|c|c|c|c|c|c|c|}
\hline \multirow[b]{2}{*}{ Source } & \multirow[b]{2}{*}{ Gas } & \multirow[b]{2}{*}{$\begin{array}{l}2019 \text { Emission Estimate } \\
\text { (MMT CO2 Eq.) }\end{array}$} & \multicolumn{4}{|c|}{$\begin{array}{l}\text { Uncertainty Range Relative to Emission Estimate } \\
\text { (MMT } \mathrm{CO}_{2} \text { Eq.) }\end{array}$} \\
\hline & & & $\begin{array}{l}\text { Lower } \\
\text { Bound }\end{array}$ & $\begin{array}{l}\text { Upper } \\
\text { Bound }\end{array}$ & $\begin{array}{l}\text { Lower } \\
\text { Bound }\end{array}$ & $\begin{array}{l}\text { Upper } \\
\text { Bound }\end{array}$ \\
\hline Non- $\mathrm{CO}_{2}$ Emissions from Forest Fires & $\mathrm{CH}_{4}$ & 0.1 & 0.1 & 0.1 & $-19.1 \%$ & $20.3 \%$ \\
\hline Non- $\mathrm{CO}_{2}$ Emissions from Forest Fires & $\mathrm{N}_{2} \mathrm{O}$ & 0.1 & 0.1 & 0.1 & $-16.9 \%$ & $17.6 \%$ \\
\hline
\end{tabular}

a These estimates include non- $\mathrm{CO}_{2}$ emissions from forest fires on Forest Land Remaining Forest Land and Land Converted to Forest Land.

${ }^{\mathrm{b}}$ Range of flux estimates predicted by Monte Carlo Stochastic Simulation for a 95 percent confidence interval, IPCC Approach 2. 


\section{Maine}

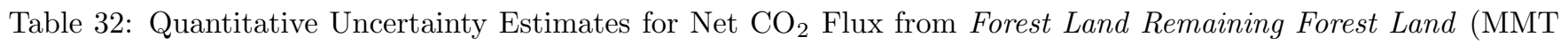
$\mathrm{CO}_{2}$ Eq. and Percent), Maine

\begin{tabular}{|c|c|c|c|c|c|}
\hline \multirow{4}{*}{ Source } & \multirow{4}{*}{$\begin{array}{l}2019 \text { Flux Estimate } \\
\text { (MMT } \mathrm{CO}_{2} \text { Eq.) }\end{array}$} & \multicolumn{4}{|c|}{ Uncertainty Range Relative to Emission Estimate } \\
\hline & & \multicolumn{2}{|c|}{ (MMT CO $\mathrm{CO}_{2}$ Eq.) } & \multicolumn{2}{|c|}{$(\%)$} \\
\hline & & Lower & Upper & Lower & Upper \\
\hline & & Bound & Bound & Bound & Bound \\
\hline Forest Ecosystem C Pools & $(9.5)$ & $(20.5)$ & 1.6 & $-116.4 \%$ & $116.4 \%$ \\
\hline
\end{tabular}




\section{Maryland}

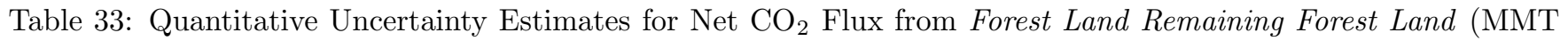
$\mathrm{CO}_{2}$ Eq. and Percent), Maryland

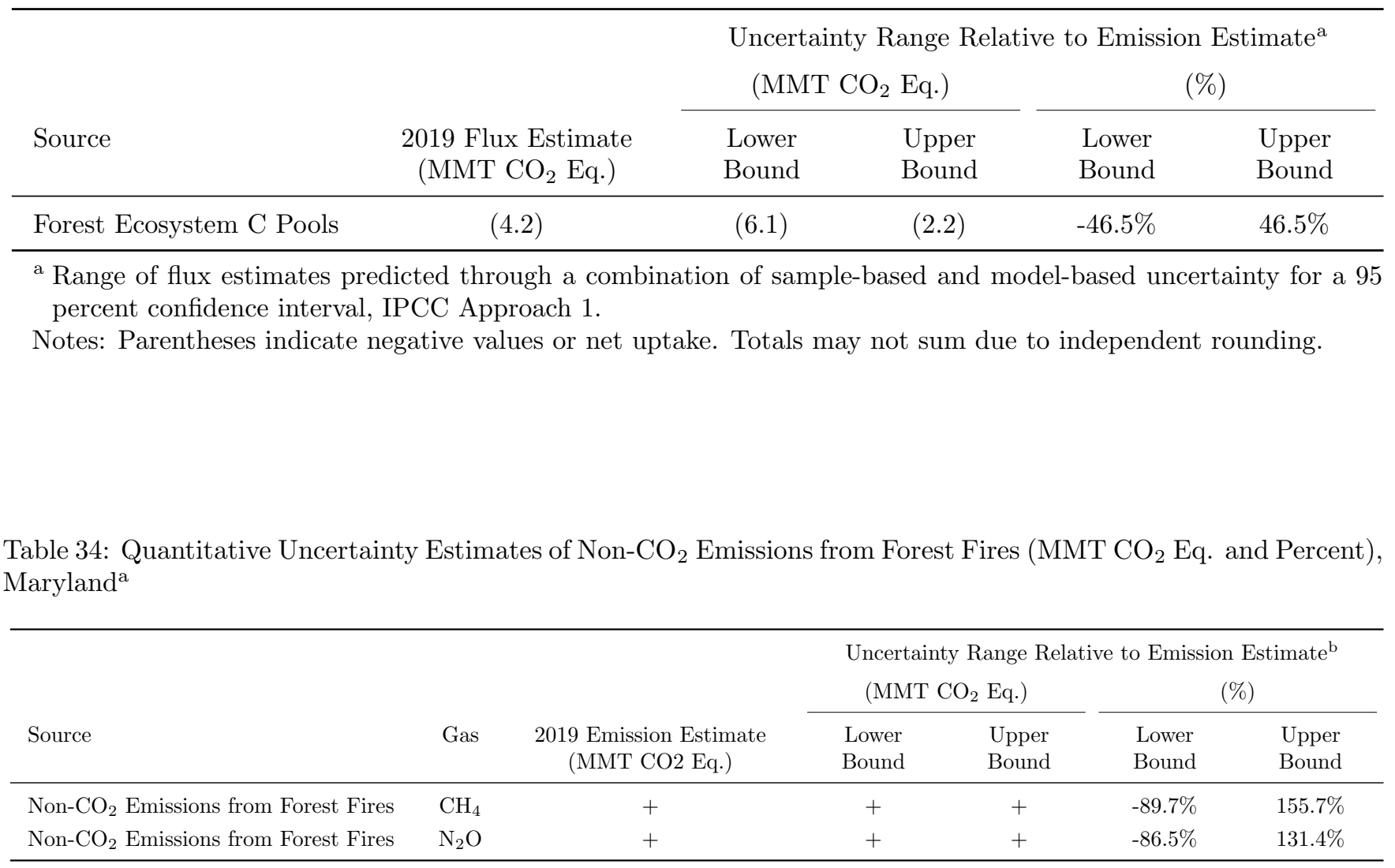

+ Absolute value does not exceed $0.05 \mathrm{MMT} \mathrm{CO}_{2} \mathrm{Eq}$.

a These estimates include non- $\mathrm{CO}_{2}$ emissions from forest fires on Forest Land Remaining Forest Land and Land Converted to Forest Land.

${ }^{\mathrm{b}}$ Range of flux estimates predicted by Monte Carlo Stochastic Simulation for a 95 percent confidence interval, IPCC Approach 2. 


\section{Massachusetts}

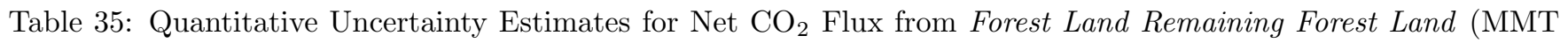
$\mathrm{CO}_{2}$ Eq. and Percent), Massachusetts

\begin{tabular}{|c|c|c|c|c|c|}
\hline \multirow{4}{*}{ Source } & \multirow{4}{*}{$\begin{array}{l}2019 \text { Flux Estimate } \\
\text { (MMT } \mathrm{CO}_{2} \text { Eq.) }\end{array}$} & \multicolumn{4}{|c|}{ Uncertainty Range Relative to Emission Estimate } \\
\hline & & \multicolumn{2}{|c|}{ (MMT CO $\mathrm{Cq}_{2}$ Eq) } & \multicolumn{2}{|c|}{$(\%)$} \\
\hline & & Lower & Upper & Lower & Upper \\
\hline & & Bound & Bound & Bound & Bound \\
\hline Forest Ecosystem C Pools & $(4.6)$ & $(6.0)$ & $(3.2)$ & $-29.9 \%$ & $29.9 \%$ \\
\hline
\end{tabular}

${ }^{a}$ Range of flux estimates predicted through a combination of sample-based and model-based uncertainty for a 95 percent confidence interval, IPCC Approach 1.

Notes: Parentheses indicate negative values or net uptake. Totals may not sum due to independent rounding. 


\section{Michigan}

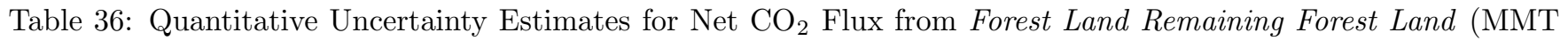
$\mathrm{CO}_{2}$ Eq. and Percent), Michigan

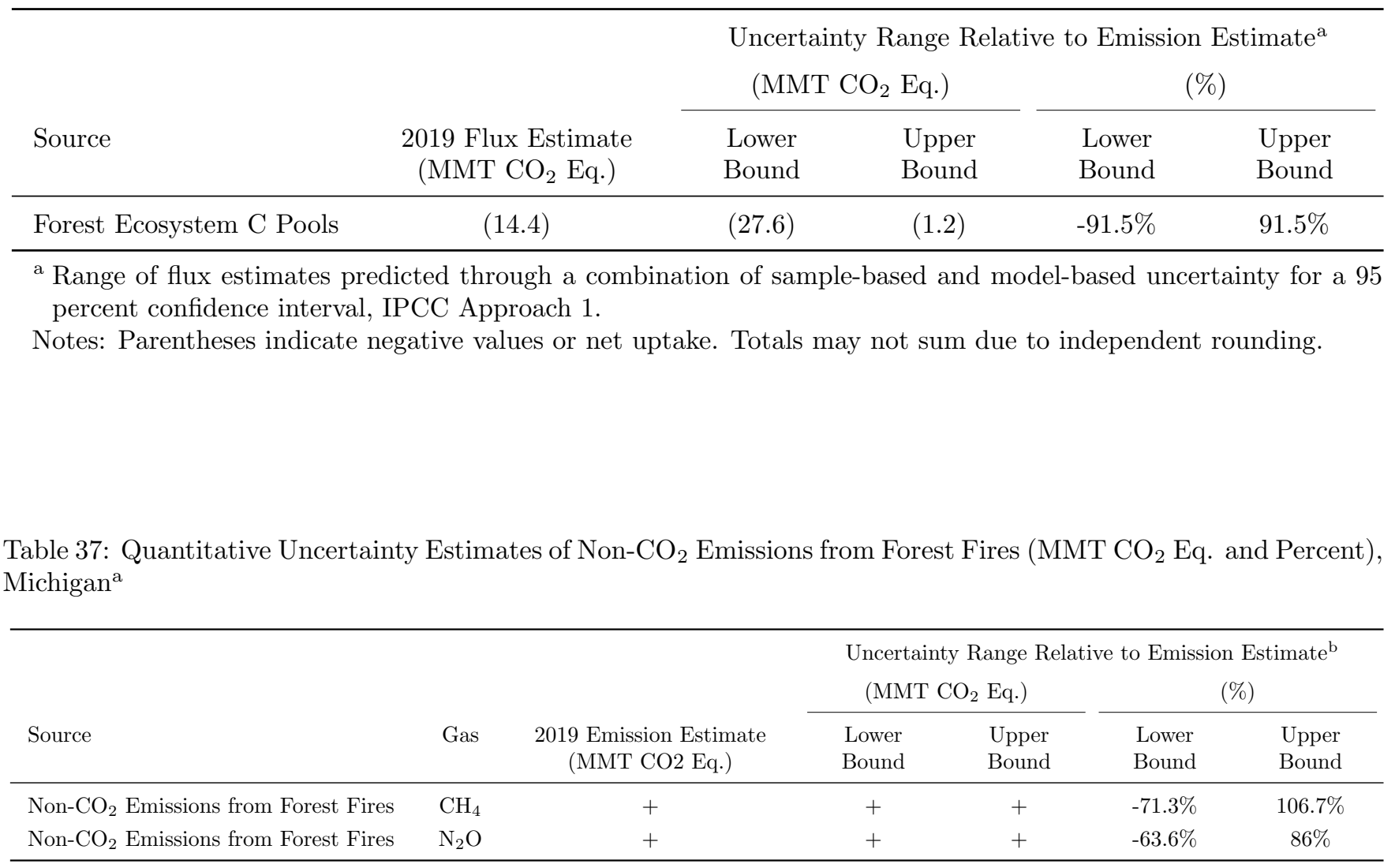

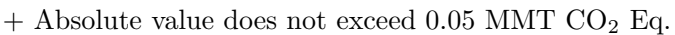

a These estimates include non- $\mathrm{CO}_{2}$ emissions from forest fires on Forest Land Remaining Forest Land and Land Converted to Forest Land.

${ }^{\mathrm{b}}$ Range of flux estimates predicted by Monte Carlo Stochastic Simulation for a 95 percent confidence interval, IPCC Approach 2. 


\section{Minnesota}

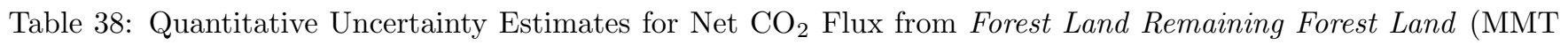
$\mathrm{CO}_{2}$ Eq. and Percent), Minnesota

\begin{tabular}{|c|c|c|c|c|c|}
\hline \multirow{4}{*}{ Source } & \multirow{4}{*}{$\begin{array}{l}2019 \text { Flux Estimate } \\
\text { (MMT } \mathrm{CO}_{2} \text { Eq.) }\end{array}$} & \multicolumn{4}{|c|}{ Uncertainty Range Relative to Emission Estimate } \\
\hline & & \multicolumn{2}{|c|}{ (MMT CO $\mathrm{CO}_{2}$ Eq.) } & \multicolumn{2}{|c|}{$(\%)$} \\
\hline & & Lower & Upper & Lower & Upper \\
\hline & & Bound & Bound & Bound & Bound \\
\hline Forest Ecosystem C Pools & $(13.4)$ & $(21.9)$ & $(5.0)$ & $-63.1 \%$ & $63.1 \%$ \\
\hline
\end{tabular}




\section{Mississippi}

Table 39: Quantitative Uncertainty Estimates for Net $\mathrm{CO}_{2}$ Flux from Forest Land Remaining Forest Land (MMT $\mathrm{CO}_{2}$ Eq. and Percent), Mississippi

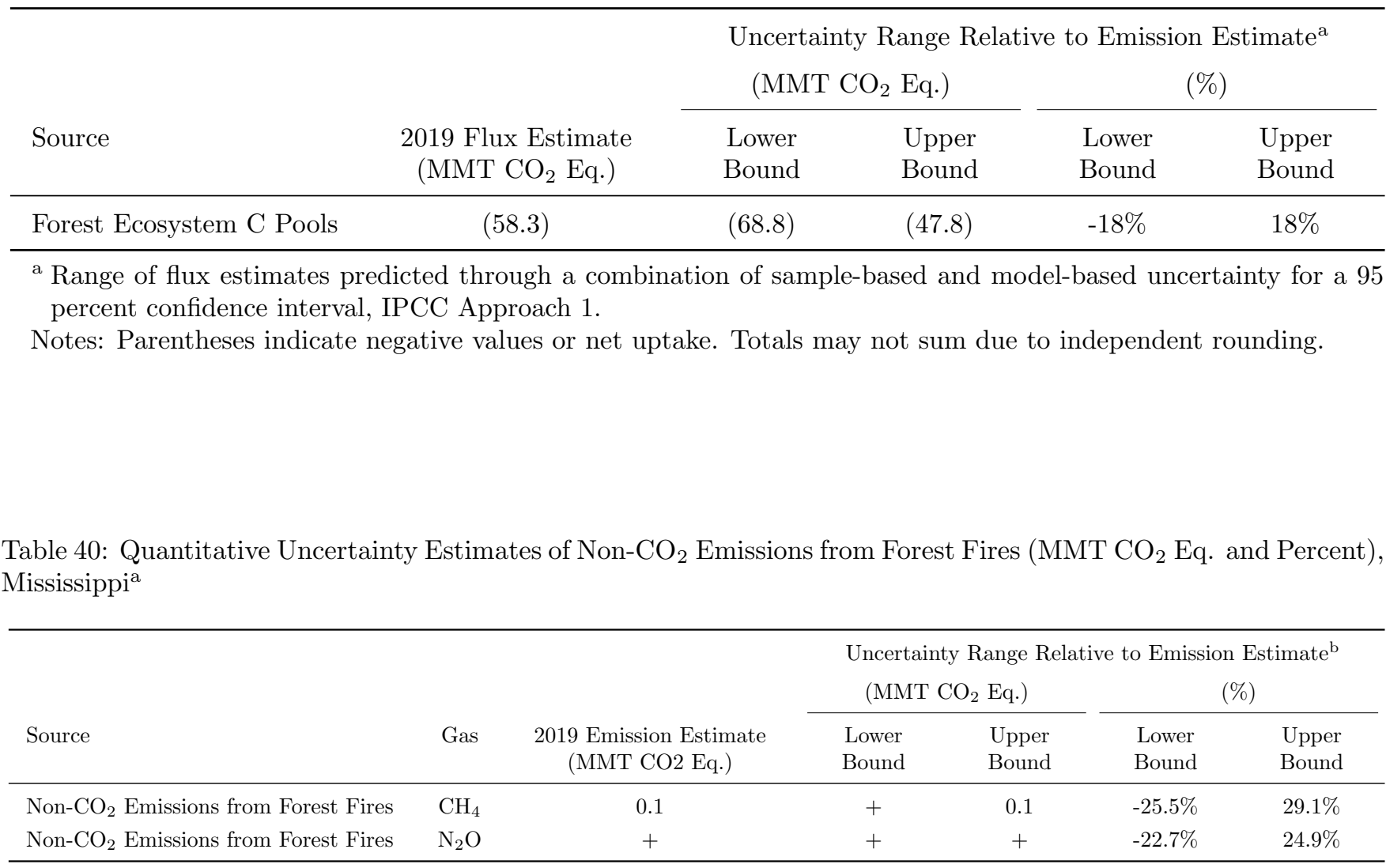

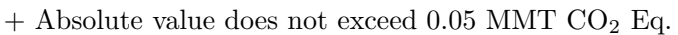

a These estimates include non- $\mathrm{CO}_{2}$ emissions from forest fires on Forest Land Remaining Forest Land and Land Converted to Forest Land.

${ }^{\mathrm{b}}$ Range of flux estimates predicted by Monte Carlo Stochastic Simulation for a 95 percent confidence interval, IPCC Approach 2. 


\section{Missouri}

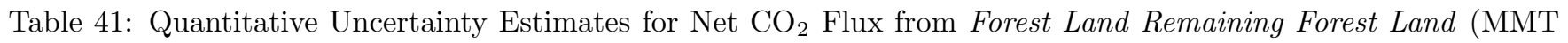
$\mathrm{CO}_{2}$ Eq. and Percent), Missouri

\begin{tabular}{|c|c|c|c|c|c|}
\hline \multirow{4}{*}{ Source } & \multirow{4}{*}{$\begin{array}{l}2019 \text { Flux Estimate } \\
\text { (MMT } \mathrm{CO}_{2} \text { Eq.) }\end{array}$} & \multicolumn{4}{|c|}{ Uncertainty Range Relative to Emission Estimate $^{a}$} \\
\hline & & \multicolumn{2}{|c|}{ (MMT $\mathrm{CO}_{2}$ Eq.) } & \multicolumn{2}{|c|}{$(\%)$} \\
\hline & & Lower & Upper & Lower & Upper \\
\hline & & Bound & Bound & Bound & Bound \\
\hline Forest Ecosystem C Pools & $(10.8)$ & $(20.2)$ & $(1.3)$ & $-87.8 \%$ & $87.8 \%$ \\
\hline
\end{tabular}

${ }^{a}$ Range of flux estimates predicted through a combination of sample-based and model-based uncertainty for a 95 percent confidence interval, IPCC Approach 1.

Notes: Parentheses indicate negative values or net uptake. Totals may not sum due to independent rounding.

Table 42: Quantitative Uncertainty Estimates of Non- $\mathrm{CO}_{2}$ Emissions from Forest Fires $\left(\mathrm{MMT} \mathrm{CO}_{2}\right.$ Eq. and Percent), Missouri $^{\mathrm{a}}$

\begin{tabular}{|c|c|c|c|c|c|c|}
\hline \multirow[b]{3}{*}{ Source } & \multirow[b]{3}{*}{ Gas } & \multirow[b]{3}{*}{$\begin{array}{l}2019 \text { Emission Estimate } \\
\text { (MMT CO2 Eq.) }\end{array}$} & \multicolumn{4}{|c|}{ Uncertainty Range Relative to Emission Estimate ${ }^{b}$} \\
\hline & & & \multicolumn{2}{|c|}{ (MMT $\mathrm{CO}_{2}$ Eq.) } & \multicolumn{2}{|c|}{$(\%)$} \\
\hline & & & $\begin{array}{l}\text { Lower } \\
\text { Bound }\end{array}$ & $\begin{array}{l}\text { Upper } \\
\text { Bound }\end{array}$ & $\begin{array}{l}\text { Lower } \\
\text { Bound }\end{array}$ & $\begin{array}{l}\text { Upper } \\
\text { Bound }\end{array}$ \\
\hline Non- $\mathrm{CO}_{2}$ Emissions from Forest Fires & $\mathrm{CH}_{4}$ & + & + & + & $-59.5 \%$ & $82 \%$ \\
\hline Non- $\mathrm{CO}_{2}$ Emissions from Forest Fires & $\mathrm{N}_{2} \mathrm{O}$ & + & + & + & $-55.6 \%$ & $68.9 \%$ \\
\hline
\end{tabular}

+ Absolute value does not exceed 0.05 MMT $\mathrm{CO}_{2} \mathrm{Eq}$.

${ }^{a}$ These estimates include non- $\mathrm{CO}_{2}$ emissions from forest fires on Forest Land Remaining Forest Land and Land Converted to Forest Land.

${ }^{\mathrm{b}}$ Range of flux estimates predicted by Monte Carlo Stochastic Simulation for a 95 percent confidence interval, IPCC Approach 2. 


\section{Montana}

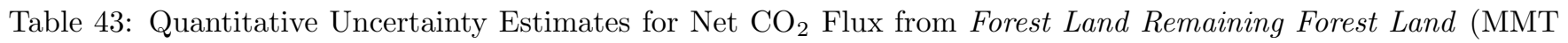
$\mathrm{CO}_{2}$ Eq. and Percent), Montana

\begin{tabular}{|c|c|c|c|c|c|}
\hline \multirow[b]{3}{*}{ Source } & \multirow[b]{3}{*}{$\begin{array}{l}2019 \text { Flux Estimate } \\
\text { (MMT } \mathrm{CO}_{2} \text { Eq.) }\end{array}$} & \multicolumn{4}{|c|}{ Uncertainty Range Relative to Emission Estimate ${ }^{a}$} \\
\hline & & \multicolumn{2}{|c|}{ (MMT CO $\mathrm{Cq}_{2}$ Eq) } & \multicolumn{2}{|c|}{$(\%)$} \\
\hline & & Lower & $\begin{array}{l}\text { Upper } \\
\text { Bound }\end{array}$ & Lower & Upper \\
\hline Forest Ecosystem C Pools & 10.4 & $(18.9)$ & 39.8 & $-280.6 \%$ & $280.6 \%$ \\
\hline
\end{tabular}

${ }^{a}$ Range of flux estimates predicted through a combination of sample-based and model-based uncertainty for a 95 percent confidence interval, IPCC Approach 1.

Notes: Parentheses indicate negative values or net uptake. Totals may not sum due to independent rounding.

Table 44: Quantitative Uncertainty Estimates of Non- $\mathrm{CO}_{2}$ Emissions from Forest Fires (MMT $\mathrm{CO}_{2}$ Eq. and Percent), Montana $^{\mathrm{a}}$

\begin{tabular}{|c|c|c|c|c|c|c|}
\hline \multirow[b]{2}{*}{ Source } & \multirow[b]{2}{*}{ Gas } & \multirow[b]{2}{*}{$\begin{array}{l}2019 \text { Emission Estimate } \\
\text { (MMT CO2 Eq.) }\end{array}$} & \multicolumn{4}{|c|}{$\begin{array}{l}\text { Uncertainty Range Relative to Emission Estimate } \\
\text { (MMT } \mathrm{CO}_{2} \text { Eq.) }\end{array}$} \\
\hline & & & $\begin{array}{l}\text { Lower } \\
\text { Bound }\end{array}$ & $\begin{array}{l}\text { Upper } \\
\text { Bound }\end{array}$ & $\begin{array}{l}\text { Lower } \\
\text { Bound }\end{array}$ & $\begin{array}{l}\text { Upper } \\
\text { Bound }\end{array}$ \\
\hline Non- $\mathrm{CO}_{2}$ Emissions from Forest Fires & $\mathrm{CH}_{4}$ & 0.2 & 0.2 & 0.3 & $-25.2 \%$ & $26.9 \%$ \\
\hline Non- $\mathrm{CO}_{2}$ Emissions from Forest Fires & $\mathrm{N}_{2} \mathrm{O}$ & 0.1 & 0.1 & 0.2 & $-19.2 \%$ & $21.2 \%$ \\
\hline
\end{tabular}

a These estimates include non- $\mathrm{CO}_{2}$ emissions from forest fires on Forest Land Remaining Forest Land and Land Converted to Forest Land.

${ }^{\mathrm{b}}$ Range of flux estimates predicted by Monte Carlo Stochastic Simulation for a 95 percent confidence interval, IPCC Approach 2. 


\section{Nebraska}

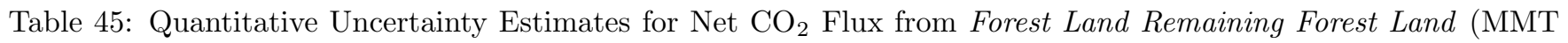
$\mathrm{CO}_{2}$ Eq. and Percent), Nebraska

\begin{tabular}{|c|c|c|c|c|c|}
\hline \multirow[b]{3}{*}{ Source } & \multirow[b]{3}{*}{$\begin{array}{l}2019 \text { Flux Estimate } \\
\text { (MMT } \mathrm{CO}_{2} \text { Eq.) }\end{array}$} & \multicolumn{4}{|c|}{ Uncertainty Range Relative to Emission Estimate } \\
\hline & & \multicolumn{2}{|c|}{ (MMT $\mathrm{CO}_{2}$ Eq.) } & \multicolumn{2}{|c|}{$(\%)$} \\
\hline & & $\begin{array}{l}\text { Lower } \\
\text { Bound }\end{array}$ & $\begin{array}{l}\text { Upper } \\
\text { Bound }\end{array}$ & $\begin{array}{l}\text { Lower } \\
\text { Bound }\end{array}$ & $\begin{array}{l}\text { Upper } \\
\text { Bound }\end{array}$ \\
\hline Forest Ecosystem C Pools & $(0.6)$ & $(0.8)$ & $(0.4)$ & $-33.1 \%$ & $33.1 \%$ \\
\hline
\end{tabular}

${ }^{a}$ Range of flux estimates predicted through a combination of sample-based and model-based uncertainty for a 95 percent confidence interval, IPCC Approach 1.

Notes: Parentheses indicate negative values or net uptake. Totals may not sum due to independent rounding.

Table 46: Quantitative Uncertainty Estimates of Non- $\mathrm{CO}_{2}$ Emissions from Forest Fires $\left(\mathrm{MMT} \mathrm{CO}_{2}\right.$ Eq. and Percent), Nebraska $^{\mathrm{a}}$

\begin{tabular}{|c|c|c|c|c|c|c|}
\hline \multirow[b]{2}{*}{ Source } & \multirow[b]{2}{*}{ Gas } & \multirow[b]{2}{*}{$\begin{array}{l}2019 \text { Emission Estimate } \\
\text { (MMT CO2 Eq.) }\end{array}$} & \multicolumn{4}{|c|}{$\begin{array}{l}\text { Uncertainty Range Relative to Emission Estimate } \\
\text { (MMT } \mathrm{CO}_{2} \text { Eq.) }\end{array}$} \\
\hline & & & $\begin{array}{l}\text { Lower } \\
\text { Bound }\end{array}$ & $\begin{array}{l}\text { Upper } \\
\text { Bound }\end{array}$ & $\begin{array}{l}\text { Lower } \\
\text { Bound }\end{array}$ & $\begin{array}{l}\text { Upper } \\
\text { Bound }\end{array}$ \\
\hline Non- $\mathrm{CO}_{2}$ Emissions from Forest Fires & $\mathrm{CH}_{4}$ & + & + & + & $-89.6 \%$ & $161.5 \%$ \\
\hline Non- $\mathrm{CO}_{2}$ Emissions from Forest Fires & $\mathrm{N}_{2} \mathrm{O}$ & + & + & + & $-86.1 \%$ & $137.3 \%$ \\
\hline
\end{tabular}

+ Absolute value does not exceed 0.05 MMT $\mathrm{CO}_{2} \mathrm{Eq}$.

a These estimates include non- $\mathrm{CO}_{2}$ emissions from forest fires on Forest Land Remaining Forest Land and Land Converted to Forest Land.

${ }^{\mathrm{b}}$ Range of flux estimates predicted by Monte Carlo Stochastic Simulation for a 95 percent confidence interval, IPCC Approach 2. 


\section{Nevada}

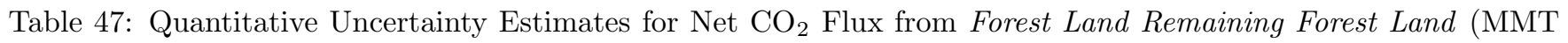
$\mathrm{CO}_{2}$ Eq. and Percent), Nevada

\begin{tabular}{|c|c|c|c|c|c|}
\hline \multirow[b]{3}{*}{ Source } & \multirow[b]{3}{*}{$\begin{array}{l}2019 \text { Flux Estimate } \\
\text { (MMT } \mathrm{CO}_{2} \text { Eq.) }\end{array}$} & \multicolumn{4}{|c|}{ Uncertainty Range Relative to Emission Estimate ${ }^{a}$} \\
\hline & & \multicolumn{2}{|c|}{ (MMT CO $\mathrm{Cq}_{2}$ Eq) } & \multicolumn{2}{|c|}{$(\%)$} \\
\hline & & Lower & Upper & Lower & Upper \\
\hline Forest Ecosystem C Pools & 0.1 & $(0.9)$ & 1.1 & $-779.9 \%$ & $779.9 \%$ \\
\hline
\end{tabular}

${ }^{a}$ Range of flux estimates predicted through a combination of sample-based and model-based uncertainty for a 95 percent confidence interval, IPCC Approach 1.

Notes: Parentheses indicate negative values or net uptake. Totals may not sum due to independent rounding.

Table 48: Quantitative Uncertainty Estimates of Non- $\mathrm{CO}_{2}$ Emissions from Forest Fires $\left(\mathrm{MMT} \mathrm{CO}_{2}\right.$ Eq. and Percent), Nevada $^{a}$

\begin{tabular}{|c|c|c|c|c|c|c|}
\hline \multirow[b]{3}{*}{ Source } & \multirow[b]{3}{*}{ Gas } & \multirow[b]{3}{*}{$\begin{array}{l}2019 \text { Emission Estimate } \\
\text { (MMT CO2 Eq.) }\end{array}$} & \multicolumn{4}{|c|}{ Uncertainty Range Relative to Emission Estimate ${ }^{\mathrm{b}}$} \\
\hline & & & \multicolumn{2}{|c|}{ (MMT $\mathrm{CO}_{2}$ Eq.) } & \multicolumn{2}{|c|}{$(\%)$} \\
\hline & & & $\begin{array}{l}\text { Lower } \\
\text { Bound }\end{array}$ & $\begin{array}{l}\text { Upper } \\
\text { Bound }\end{array}$ & $\begin{array}{l}\text { Lower } \\
\text { Bound }\end{array}$ & $\begin{array}{l}\text { Upper } \\
\text { Bound }\end{array}$ \\
\hline Non- $\mathrm{CO}_{2}$ Emissions from Forest Fires & $\mathrm{CH}_{4}$ & 0.1 & 0.1 & 0.1 & $-38.4 \%$ & $44.8 \%$ \\
\hline Non- $\mathrm{CO}_{2}$ Emissions from Forest Fires & $\mathrm{N}_{2} \mathrm{O}$ & 0.1 & + & 0.1 & $-29.4 \%$ & $34.7 \%$ \\
\hline
\end{tabular}

+ Absolute value does not exceed 0.05 MMT $\mathrm{CO}_{2} \mathrm{Eq}$.

a These estimates include non- $\mathrm{CO}_{2}$ emissions from forest fires on Forest Land Remaining Forest Land and Land Converted to Forest Land.

${ }^{\mathrm{b}}$ Range of flux estimates predicted by Monte Carlo Stochastic Simulation for a 95 percent confidence interval, IPCC Approach 2. 


\section{New Hampshire}

Table 49: Quantitative Uncertainty Estimates for Net $\mathrm{CO}_{2}$ Flux from Forest Land Remaining Forest Land (MMT $\mathrm{CO}_{2}$ Eq. and Percent), New Hampshire

\begin{tabular}{|c|c|c|c|c|c|}
\hline \multirow{4}{*}{ Source } & \multirow{4}{*}{$\begin{array}{l}2019 \text { Flux Estimate } \\
\text { (MMT } \mathrm{CO}_{2} \text { Eq.) }\end{array}$} & \multicolumn{4}{|c|}{ Uncertainty Range Relative to Emission Estimate } \\
\hline & & \multicolumn{2}{|c|}{ (MMT CO 2 Eq.) } & \multicolumn{2}{|c|}{$(\%)$} \\
\hline & & Lower & Upper & Lower & Upper \\
\hline & & Bound & Bound & Bound & Bound \\
\hline Forest Ecosystem C Pools & $(5.3)$ & $(7.5)$ & $(3.1)$ & $-41.7 \%$ & $41.7 \%$ \\
\hline
\end{tabular}




\section{New Jersey}

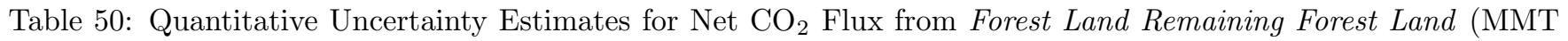
$\mathrm{CO}_{2}$ Eq. and Percent), New Jersey

\begin{tabular}{|c|c|c|c|c|c|}
\hline \multirow{4}{*}{ Source } & \multirow{4}{*}{$\begin{array}{l}2019 \text { Flux Estimate } \\
\text { (MMT CO } \mathrm{CO}_{2} \text { Eq.) }\end{array}$} & \multicolumn{4}{|c|}{ Uncertainty Range Relative to Emission Estimate ${ }^{a}$} \\
\hline & & \multicolumn{2}{|c|}{$(\mathrm{MMT} \mathrm{CO} 2$ Eq.) } & \multicolumn{2}{|c|}{$(\%)$} \\
\hline & & Lower & Upper & Lower & Upper \\
\hline & & Bound & Bound & Bound & Bound \\
\hline Forest Ecosystem C Pools & $(2.1)$ & $(2.4)$ & $(1.8)$ & $-15.5 \%$ & $15.5 \%$ \\
\hline
\end{tabular}

Table 51: Quantitative Uncertainty Estimates of Non- $\mathrm{CO}_{2}$ Emissions from Forest Fires $\left(\mathrm{MMT} \mathrm{CO}_{2}\right.$ Eq. and Percent), New Jersey ${ }^{\mathrm{a}}$

\begin{tabular}{|c|c|c|c|c|c|c|}
\hline \multirow[b]{2}{*}{ Source } & \multirow[b]{2}{*}{ Gas } & \multirow[b]{2}{*}{$\begin{array}{l}2019 \text { Emission Estimate } \\
\text { (MMT CO2 Eq.) }\end{array}$} & $\begin{array}{r}\text { Uncert } \\
(\mathrm{MM}\end{array}$ & $\begin{array}{l}\text { ange Re } \\
\text { Eq.) }\end{array}$ & to Emissi & timate $^{\mathrm{b}}$ \\
\hline & & & $\begin{array}{l}\text { Lower } \\
\text { Bound }\end{array}$ & $\begin{array}{l}\text { Upper } \\
\text { Bound }\end{array}$ & $\begin{array}{l}\text { Lower } \\
\text { Bound }\end{array}$ & $\begin{array}{l}\text { Upper } \\
\text { Bound }\end{array}$ \\
\hline Non- $\mathrm{CO}_{2}$ Emissions from Forest Fires & $\mathrm{CH}_{4}$ & + & + & + & $-74.9 \%$ & $115 \%$ \\
\hline Non- $\mathrm{CO}_{2}$ Emissions from Forest Fires & $\mathrm{N}_{2} \mathrm{O}$ & + & + & + & $-71.1 \%$ & $96.6 \%$ \\
\hline
\end{tabular}

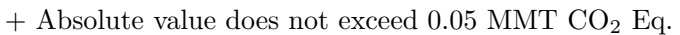

a These estimates include non- $\mathrm{CO}_{2}$ emissions from forest fires on Forest Land Remaining Forest Land and Land Converted to Forest Land.

${ }^{\mathrm{b}}$ Range of flux estimates predicted by Monte Carlo Stochastic Simulation for a 95 percent confidence interval, IPCC Approach 2. 


\section{New Mexico}

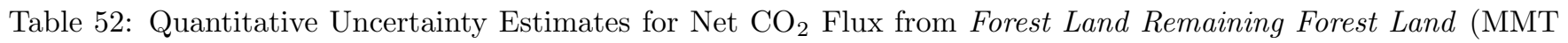
$\mathrm{CO}_{2}$ Eq. and Percent), New Mexico

\begin{tabular}{|c|c|c|c|c|c|}
\hline \multirow[b]{2}{*}{ Source } & \multirow[b]{2}{*}{$\begin{array}{l}2019 \text { Flux Estimate } \\
\text { (MMT } \mathrm{CO}_{2} \text { Eq.) }\end{array}$} & $\begin{array}{r}\text { Uncert } \\
(\mathrm{MM}\end{array}$ & $\begin{array}{l}\text { (ange Re } \\
\text { Eq.) }\end{array}$ & to Emissi & timate $^{\mathrm{a}}$ \\
\hline & & $\begin{array}{l}\text { Lower } \\
\text { Bound }\end{array}$ & $\begin{array}{l}\text { Upper } \\
\text { Bound }\end{array}$ & $\begin{array}{l}\text { Lower } \\
\text { Bound }\end{array}$ & $\begin{array}{l}\text { Upper } \\
\text { Bound }\end{array}$ \\
\hline Forest Ecosystem C Pools & 3.8 & $(3.2)$ & 10.9 & $-183.4 \%$ & $183.4 \%$ \\
\hline
\end{tabular}

Table 53: Quantitative Uncertainty Estimates of Non- $\mathrm{CO}_{2}$ Emissions from Forest Fires (MMT $\mathrm{CO}_{2}$ Eq. and Percent), New Mexico ${ }^{\mathrm{a}}$

\begin{tabular}{|c|c|c|c|c|c|c|}
\hline \multirow[b]{3}{*}{ Source } & \multirow[b]{3}{*}{ Gas } & \multirow[b]{3}{*}{$\begin{array}{l}2019 \text { Emission Estimate } \\
\text { (MMT CO2 Eq.) }\end{array}$} & \multicolumn{4}{|c|}{ Uncertainty Range Relative to Emission Estimate ${ }^{\mathrm{b}}$} \\
\hline & & & \multicolumn{2}{|c|}{ (MMT $\mathrm{CO}_{2}$ Eq.) } & \multicolumn{2}{|c|}{$(\%)$} \\
\hline & & & $\begin{array}{l}\text { Lower } \\
\text { Bound }\end{array}$ & $\begin{array}{l}\text { Upper } \\
\text { Bound }\end{array}$ & $\begin{array}{l}\text { Lower } \\
\text { Bound }\end{array}$ & $\begin{array}{l}\text { Upper } \\
\text { Bound }\end{array}$ \\
\hline Non- $\mathrm{CO}_{2}$ Emissions from Forest Fires & $\mathrm{CH}_{4}$ & 0.2 & 0.1 & 0.3 & $-43.1 \%$ & $53.7 \%$ \\
\hline Non- $\mathrm{CO}_{2}$ Emissions from Forest Fires & $\mathrm{N}_{2} \mathrm{O}$ & 0.1 & 0.1 & 0.2 & $-34.2 \%$ & $41.6 \%$ \\
\hline
\end{tabular}

a These estimates include non- $\mathrm{CO}_{2}$ emissions from forest fires on Forest Land Remaining Forest Land and Land Converted to Forest Land.

${ }^{\mathrm{b}}$ Range of flux estimates predicted by Monte Carlo Stochastic Simulation for a 95 percent confidence interval, IPCC Approach 2. 


\section{New York}

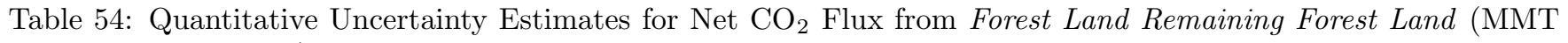
$\mathrm{CO}_{2}$ Eq. and Percent), New York

\begin{tabular}{|c|c|c|c|c|c|}
\hline \multirow[b]{3}{*}{ Source } & \multirow[b]{3}{*}{$\begin{array}{l}2019 \text { Flux Estimate } \\
\text { (MMT } \mathrm{CO}_{2} \text { Eq.) }\end{array}$} & \multicolumn{4}{|c|}{ Uncertainty Range Relative to Emission Estimate } \\
\hline & & \multicolumn{2}{|c|}{ (MMT CO $\mathrm{Cq}_{2}$ Eq.) } & \multicolumn{2}{|c|}{$(\%)$} \\
\hline & & Lower & Upper & Lower & Upper \\
\hline Forest Ecosystem C Pools & $(23.9)$ & $(32.4)$ & $(15.5)$ & $-35.3 \%$ & $35.3 \%$ \\
\hline
\end{tabular}

${ }^{a}$ Range of flux estimates predicted through a combination of sample-based and model-based uncertainty for a 95 percent confidence interval, IPCC Approach 1.

Notes: Parentheses indicate negative values or net uptake. Totals may not sum due to independent rounding. 


\section{North Carolina}

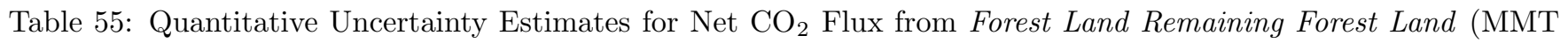
$\mathrm{CO}_{2}$ Eq. and Percent), North Carolina

\begin{tabular}{|c|c|c|c|c|c|}
\hline \multirow[b]{3}{*}{ Source } & \multirow{3}{*}{$\begin{array}{l}2019 \text { Flux Estimate } \\
\text { (MMT } \mathrm{CO}_{2} \text { Eq.) }\end{array}$} & \multicolumn{4}{|c|}{ Uncertainty Range Relative to Emission Estimate $^{a}$} \\
\hline & & \multicolumn{2}{|c|}{ (MMT $\mathrm{CO}_{2}$ Eq.) } & \multicolumn{2}{|c|}{$(\%)$} \\
\hline & & Lower & Upper & Lower & Upper \\
\hline Forest Ecosystem C Pools & $(30.3)$ & $(34.9)$ & $(25.7)$ & $-15.2 \%$ & $15.2 \%$ \\
\hline
\end{tabular}

${ }^{a}$ Range of flux estimates predicted through a combination of sample-based and model-based uncertainty for a 95 percent confidence interval, IPCC Approach 1.

Notes: Parentheses indicate negative values or net uptake. Totals may not sum due to independent rounding.

Table 56: Quantitative Uncertainty Estimates of Non- $\mathrm{CO}_{2}$ Emissions from Forest Fires $\left(\mathrm{MMT} \mathrm{CO}_{2}\right.$ Eq. and Percent), North Carolina ${ }^{a}$

\begin{tabular}{|c|c|c|c|c|c|c|}
\hline \multirow[b]{3}{*}{ Source } & \multirow[b]{3}{*}{ Gas } & \multirow[b]{3}{*}{$\begin{array}{l}2019 \text { Emission Estimate } \\
\text { (MMT CO2 Eq.) }\end{array}$} & \multicolumn{4}{|c|}{ Uncertainty Range Relative to Emission Estimate ${ }^{b}$} \\
\hline & & & \multicolumn{2}{|c|}{ (MMT $\mathrm{CO}_{2}$ Eq.) } & \multicolumn{2}{|c|}{$(\%)$} \\
\hline & & & $\begin{array}{l}\text { Lower } \\
\text { Bound }\end{array}$ & $\begin{array}{l}\text { Upper } \\
\text { Bound }\end{array}$ & $\begin{array}{l}\text { Lower } \\
\text { Bound }\end{array}$ & $\begin{array}{l}\text { Upper } \\
\text { Bound }\end{array}$ \\
\hline Non- $\mathrm{CO}_{2}$ Emissions from Forest Fires & $\mathrm{CH}_{4}$ & + & + & + & $-44.5 \%$ & $58 \%$ \\
\hline Non- $\mathrm{CO}_{2}$ Emissions from Forest Fires & $\mathrm{N}_{2} \mathrm{O}$ & + & + & + & $-40.4 \%$ & $48.8 \%$ \\
\hline
\end{tabular}

+ Absolute value does not exceed 0.05 MMT $\mathrm{CO}_{2} \mathrm{Eq}$.

${ }^{a}$ These estimates include non- $\mathrm{CO}_{2}$ emissions from forest fires on Forest Land Remaining Forest Land and Land Converted to Forest Land.

${ }^{\mathrm{b}}$ Range of flux estimates predicted by Monte Carlo Stochastic Simulation for a 95 percent confidence interval, IPCC Approach 2. 


\section{North Dakota}

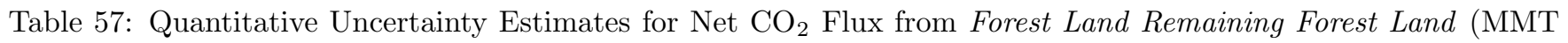
$\mathrm{CO}_{2}$ Eq. and Percent), North Dakota

\begin{tabular}{|c|c|c|c|c|c|}
\hline \multirow[b]{2}{*}{ Source } & \multirow[b]{2}{*}{$\begin{array}{l}2019 \text { Flux Estimate } \\
\text { (MMT } \mathrm{CO}_{2} \text { Eq.) }\end{array}$} & $\begin{array}{r}\text { Uncert } \\
(\mathrm{MM}\end{array}$ & $\begin{array}{l}\text { (ange Re } \\
\text { Eq.) }\end{array}$ & to Emissi & timate $^{\mathrm{a}}$ \\
\hline & & $\begin{array}{l}\text { Lower } \\
\text { Bound }\end{array}$ & $\begin{array}{l}\text { Upper } \\
\text { Bound }\end{array}$ & $\begin{array}{l}\text { Lower } \\
\text { Bound }\end{array}$ & $\begin{array}{l}\text { Upper } \\
\text { Bound }\end{array}$ \\
\hline Forest Ecosystem C Pools & $(0.1)$ & $(0.4)$ & 0.3 & $-448.8 \%$ & $448.8 \%$ \\
\hline
\end{tabular}

Table 58: Quantitative Uncertainty Estimates of Non- $\mathrm{CO}_{2}$ Emissions from Forest Fires $\left(\mathrm{MMT} \mathrm{CO}_{2}\right.$ Eq. and Percent), North Dakota ${ }^{a}$

\begin{tabular}{|c|c|c|c|c|c|c|}
\hline \multirow{4}{*}{ Source } & \multirow{4}{*}{ Gas } & \multirow{4}{*}{$\begin{array}{l}2019 \text { Emission Estimate } \\
\text { (MMT CO2 Eq.) }\end{array}$} & \multicolumn{4}{|c|}{ Uncertainty Range Relative to Emission Estimate ${ }^{b}$} \\
\hline & & & \multicolumn{2}{|c|}{ (MMT $\mathrm{CO}_{2}$ Eq.) } & \multicolumn{2}{|c|}{$(\%)$} \\
\hline & & & Lower & Upper & Lower & Upper \\
\hline & & & Bound & Bound & Bound & Bound \\
\hline Non- $\mathrm{CO}_{2}$ Emissions from Forest Fires & $\mathrm{CH}_{4}$ & + & + & + & $-79.3 \%$ & $104.4 \%$ \\
\hline Non- $\mathrm{CO}_{2}$ Emissions from Forest Fires & $\mathrm{N}_{2} \mathrm{O}$ & + & + & + & $-60.9 \%$ & $79.8 \%$ \\
\hline
\end{tabular}




\section{Ohio}

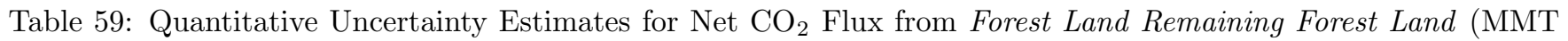
$\mathrm{CO}_{2}$ Eq. and Percent), Ohio

\begin{tabular}{|c|c|c|c|c|c|}
\hline \multirow[b]{3}{*}{ Source } & \multirow[b]{3}{*}{$\begin{array}{l}2019 \text { Flux Estimate } \\
\text { (MMT } \mathrm{CO}_{2} \text { Eq.) }\end{array}$} & \multicolumn{4}{|c|}{ Uncertainty Range Relative to Emission Estimate $^{a}$} \\
\hline & & \multicolumn{2}{|c|}{ (MMT CO $\mathrm{Cq}_{2}$ E.) } & \multicolumn{2}{|c|}{$(\%)$} \\
\hline & & $\begin{array}{l}\text { Lower } \\
\text { Bound }\end{array}$ & Upper & Lower & Upper \\
\hline Forest Ecosystem C Pools & $(6.2)$ & $(13.8)$ & 1.4 & $-122.5 \%$ & $122.5 \%$ \\
\hline
\end{tabular}




\section{Oklahoma}

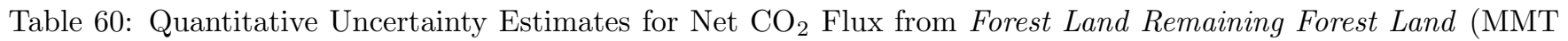
$\mathrm{CO}_{2}$ Eq. and Percent), Oklahoma

\begin{tabular}{|c|c|c|c|c|c|}
\hline \multirow[b]{3}{*}{ Source } & \multirow{3}{*}{$\begin{array}{l}2019 \text { Flux Estimate } \\
\text { (MMT } \mathrm{CO}_{2} \text { Eq.) }\end{array}$} & \multicolumn{4}{|c|}{ Uncertainty Range Relative to Emission Estimate $^{a}$} \\
\hline & & \multicolumn{2}{|c|}{ (MMT $\mathrm{CO}_{2}$ Eq.) } & \multicolumn{2}{|c|}{$(\%)$} \\
\hline & & Lower & Upper & Lower & Upper \\
\hline Forest Ecosystem C Pools & $(5.3)$ & $(7.6)$ & $(3.0)$ & $-43.6 \%$ & $43.6 \%$ \\
\hline
\end{tabular}

${ }^{a}$ Range of flux estimates predicted through a combination of sample-based and model-based uncertainty for a 95 percent confidence interval, IPCC Approach 1.

Notes: Parentheses indicate negative values or net uptake. Totals may not sum due to independent rounding.

Table 61: Quantitative Uncertainty Estimates of Non- $\mathrm{CO}_{2}$ Emissions from Forest Fires $\left(\mathrm{MMT} \mathrm{CO}_{2}\right.$ Eq. and Percent), Oklahoma $^{\mathrm{a}}$

\begin{tabular}{|c|c|c|c|c|c|c|}
\hline \multirow[b]{2}{*}{ Source } & \multirow[b]{2}{*}{ Gas } & \multirow[b]{2}{*}{$\begin{array}{l}2019 \text { Emission Estimate } \\
\text { (MMT CO2 Eq.) }\end{array}$} & \multicolumn{4}{|c|}{$\begin{array}{l}\text { Uncertainty Range Relative to Emission Estimate } \\
\left(\mathrm{MMT} \mathrm{CO}_{2} \text { Eq.) }\right.\end{array}$} \\
\hline & & & $\begin{array}{l}\text { Lower } \\
\text { Bound }\end{array}$ & $\begin{array}{l}\text { Upper } \\
\text { Bound }\end{array}$ & $\begin{array}{l}\text { Lower } \\
\text { Bound }\end{array}$ & $\begin{array}{l}\text { Upper } \\
\text { Bound }\end{array}$ \\
\hline Non- $\mathrm{CO}_{2}$ Emissions from Forest Fires & $\mathrm{CH}_{4}$ & + & + & + & $-77.4 \%$ & $124.2 \%$ \\
\hline Non- $\mathrm{CO}_{2}$ Emissions from Forest Fires & $\mathrm{N}_{2} \mathrm{O}$ & + & + & + & $-66.8 \%$ & $99 \%$ \\
\hline
\end{tabular}




\section{Oregon}

Table 62: Quantitative Uncertainty Estimates for Net $\mathrm{CO}_{2}$ Flux from Forest Land Remaining Forest Land (MMT $\mathrm{CO}_{2}$ Eq. and Percent), Oregon

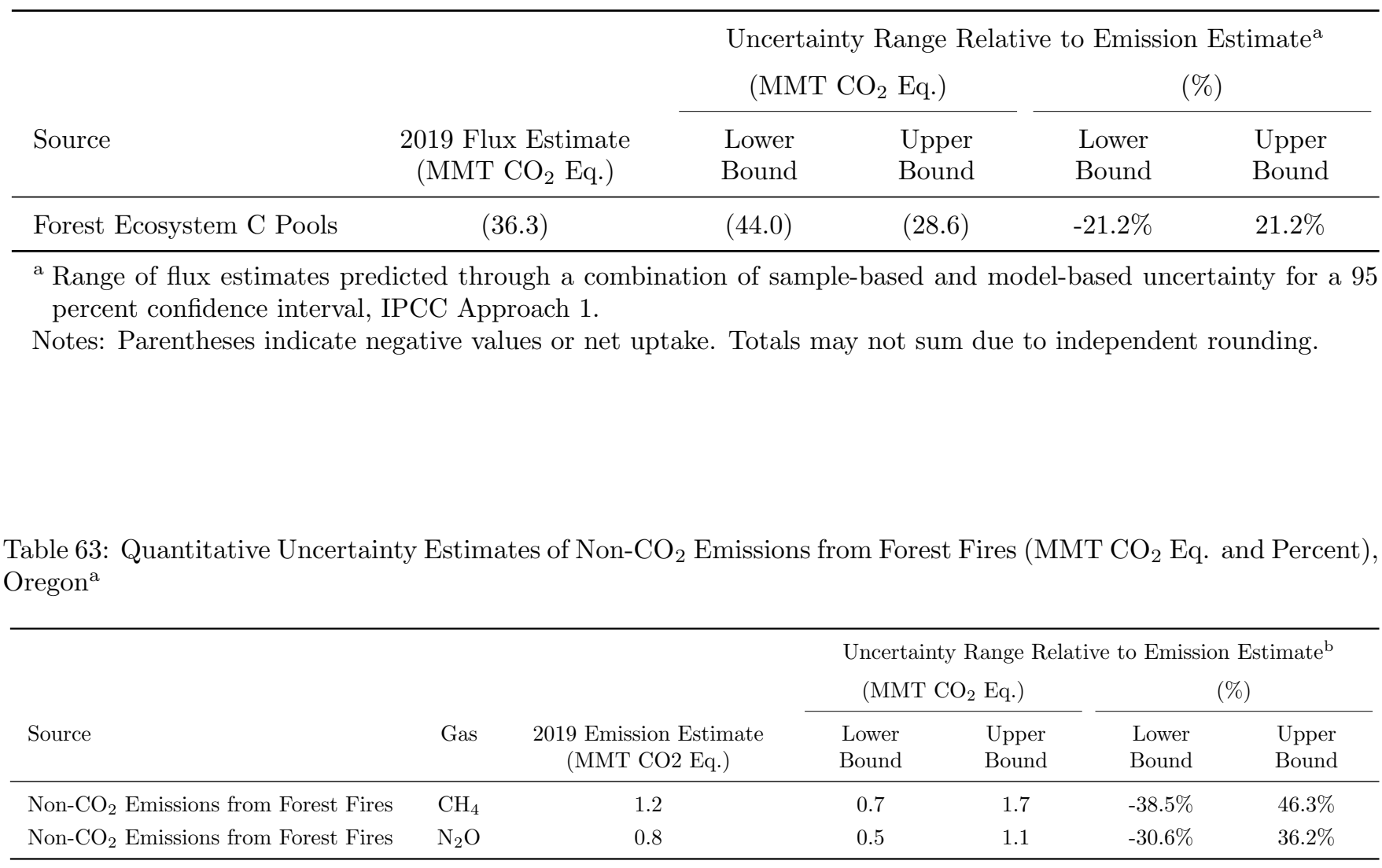

${ }^{a}$ These estimates include non- $\mathrm{CO}_{2}$ emissions from forest fires on Forest Land Remaining Forest Land and Land Converted to Forest Land.

${ }^{\mathrm{b}}$ Range of flux estimates predicted by Monte Carlo Stochastic Simulation for a 95 percent confidence interval, IPCC Approach 2. 


\section{Pennsylvania}

Table 64: Quantitative Uncertainty Estimates for Net $\mathrm{CO}_{2}$ Flux from Forest Land Remaining Forest Land (MMT $\mathrm{CO}_{2}$ Eq. and Percent), Pennsylvania

\begin{tabular}{|c|c|c|c|c|c|}
\hline \multirow[b]{2}{*}{ Source } & \multirow[b]{2}{*}{$\begin{array}{l}2019 \text { Flux Estimate } \\
\text { (MMT CO } \mathrm{CO}_{2} \text { Eq.) }\end{array}$} & $\begin{array}{r}\text { Uncert } \\
\text { (MM }\end{array}$ & \multicolumn{3}{|c|}{ Uncertainty Range Relative to Emission Estimate ${ }^{a}$} \\
\hline & & $\begin{array}{l}\text { Lower } \\
\text { Bound }\end{array}$ & $\begin{array}{l}\text { Upper } \\
\text { Bound }\end{array}$ & $\begin{array}{l}\text { Lower } \\
\text { Bound }\end{array}$ & $\begin{array}{l}\text { Upper } \\
\text { Bound }\end{array}$ \\
\hline Forest Ecosystem C Pools & $(19.5)$ & $(35.8)$ & $(3.1)$ & $-84 \%$ & $84 \%$ \\
\hline
\end{tabular}




\section{Rhode Island}

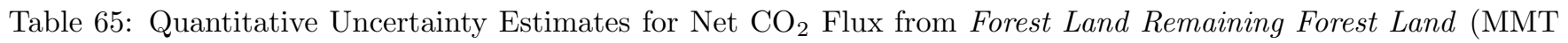
$\mathrm{CO}_{2}$ Eq. and Percent), Rhode Island

\begin{tabular}{|c|c|c|c|c|c|}
\hline \multirow[b]{3}{*}{ Source } & \multirow[b]{3}{*}{$\begin{array}{l}2019 \text { Flux Estimate } \\
\text { (MMT CO } \mathrm{CO}_{2} \text { Eq.) }\end{array}$} & \multicolumn{4}{|c|}{ Uncertainty Range Relative to Emission Estimate $^{a}$} \\
\hline & & \multicolumn{2}{|c|}{ (MMT CO 2 Eq.) } & \multicolumn{2}{|c|}{$(\%)$} \\
\hline & & $\begin{array}{l}\text { Lower } \\
\text { Bound }\end{array}$ & $\begin{array}{l}\text { Upper } \\
\text { Bound }\end{array}$ & $\begin{array}{l}\text { Lower } \\
\text { Bound }\end{array}$ & $\begin{array}{l}\text { Upper } \\
\text { Bound }\end{array}$ \\
\hline Forest Ecosystem C Pools & $(0.3)$ & $(0.8)$ & 0.2 & $-168.5 \%$ & $168.5 \%$ \\
\hline
\end{tabular}




\section{South Carolina}

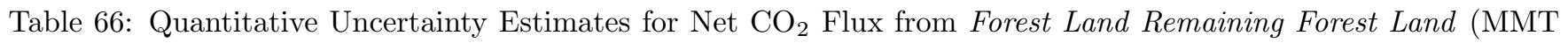
$\mathrm{CO}_{2}$ Eq. and Percent), South Carolina

\begin{tabular}{|c|c|c|c|c|c|}
\hline \multirow[b]{3}{*}{ Source } & \multirow{3}{*}{$\begin{array}{l}2019 \text { Flux Estimate } \\
\left(\mathrm{MMT} \mathrm{CO}_{2} \text { Eq.) }\right.\end{array}$} & \multicolumn{4}{|c|}{ Uncertainty Range Relative to Emission Estimate $^{a}$} \\
\hline & & \multicolumn{2}{|c|}{ (MMT CO $\mathrm{CO}_{2}$ Eq.) } & \multicolumn{2}{|c|}{$(\%)$} \\
\hline & & Lower & Upper & Lower & Upper \\
\hline Forest Ecosystem C Pools & $(12.5)$ & $(14.5)$ & $(10.5)$ & $-15.9 \%$ & $15.9 \%$ \\
\hline
\end{tabular}

${ }^{a}$ Range of flux estimates predicted through a combination of sample-based and model-based uncertainty for a 95 percent confidence interval, IPCC Approach 1.

Notes: Parentheses indicate negative values or net uptake. Totals may not sum due to independent rounding.

Table 67: Quantitative Uncertainty Estimates of Non- $\mathrm{CO}_{2}$ Emissions from Forest Fires (MMT $\mathrm{CO}_{2}$ Eq. and Percent), South Carolina ${ }^{\mathrm{a}}$

\begin{tabular}{|c|c|c|c|c|c|c|}
\hline \multirow[b]{2}{*}{ Source } & \multirow[b]{2}{*}{ Gas } & \multirow[b]{2}{*}{$\begin{array}{l}2019 \text { Emission Estimate } \\
\text { (MMT CO2 Eq.) }\end{array}$} & \multicolumn{4}{|c|}{$\begin{array}{l}\text { Uncertainty Range Relative to Emission Estimate } \\
\left(\mathrm{MMT} \mathrm{CO}_{2} \text { Eq.) }\right.\end{array}$} \\
\hline & & & $\begin{array}{l}\text { Lower } \\
\text { Bound }\end{array}$ & $\begin{array}{l}\text { Upper } \\
\text { Bound }\end{array}$ & $\begin{array}{l}\text { Lower } \\
\text { Bound }\end{array}$ & $\begin{array}{l}\text { Upper } \\
\text { Bound }\end{array}$ \\
\hline Non- $\mathrm{CO}_{2}$ Emissions from Forest Fires & $\mathrm{CH}_{4}$ & 0.1 & + & 0.1 & $-24.5 \%$ & $26.9 \%$ \\
\hline Non- $\mathrm{CO}_{2}$ Emissions from Forest Fires & $\mathrm{N}_{2} \mathrm{O}$ & + & + & + & $-21.4 \%$ & $24 \%$ \\
\hline
\end{tabular}




\section{South Dakota}

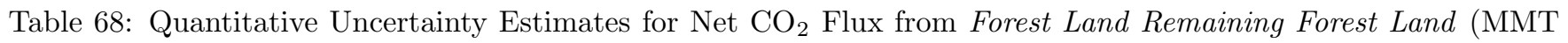
$\mathrm{CO}_{2}$ Eq. and Percent), South Dakota

\begin{tabular}{|c|c|c|c|c|c|}
\hline \multirow[b]{3}{*}{ Source } & \multirow{3}{*}{$\begin{array}{l}2019 \text { Flux Estimate } \\
\text { (MMT } \mathrm{CO}_{2} \text { Eq.) }\end{array}$} & \multicolumn{4}{|c|}{ Uncertainty Range Relative to Emission Estimate $^{a}$} \\
\hline & & \multicolumn{2}{|c|}{ (MMT CO $\mathrm{CO}_{2}$ Eq.) } & \multicolumn{2}{|c|}{$(\%)$} \\
\hline & & Lower & Upper & Lower & Upper \\
\hline Forest Ecosystem C Pools & 0.4 & $(0.8)$ & 1.7 & $-279.5 \%$ & $279.5 \%$ \\
\hline
\end{tabular}

${ }^{a}$ Range of flux estimates predicted through a combination of sample-based and model-based uncertainty for a 95 percent confidence interval, IPCC Approach 1.

Notes: Parentheses indicate negative values or net uptake. Totals may not sum due to independent rounding.

Table 69: Quantitative Uncertainty Estimates of Non- $\mathrm{CO}_{2}$ Emissions from Forest Fires $\left(\mathrm{MMT} \mathrm{CO}_{2}\right.$ Eq. and Percent), South Dakotaa

\begin{tabular}{|c|c|c|c|c|c|c|}
\hline \multirow{4}{*}{ Source } & \multirow{4}{*}{ Gas } & \multirow{4}{*}{$\begin{array}{l}2019 \text { Emission Estimate } \\
\text { (MMT CO2 Eq.) }\end{array}$} & \multicolumn{4}{|c|}{ Uncertainty Range Relative to Emission Estimate ${ }^{\mathrm{b}}$} \\
\hline & & & \multicolumn{2}{|c|}{ (MMT $\mathrm{CO}_{2}$ Eq.) } & \multicolumn{2}{|c|}{$(\%)$} \\
\hline & & & Lower & Upper & Lower & Upper \\
\hline & & & Bound & Bound & Bound & Bound \\
\hline Non- $\mathrm{CO}_{2}$ Emissions from Forest Fires & $\mathrm{CH}_{4}$ & 0.1 & + & 0.1 & $-75.6 \%$ & $100.5 \%$ \\
\hline Non- $\mathrm{CO}_{2}$ Emissions from Forest Fires & $\mathrm{N}_{2} \mathrm{O}$ & + & + & 0.1 & $-57 \%$ & $75.2 \%$ \\
\hline
\end{tabular}




\section{Tennessee}

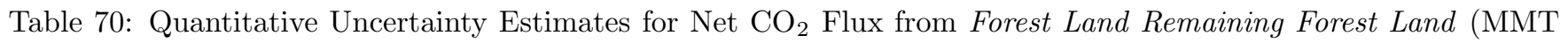
$\mathrm{CO}_{2}$ Eq. and Percent), Tennessee

\begin{tabular}{|c|c|c|c|c|c|}
\hline \multirow[b]{3}{*}{ Source } & \multirow[b]{3}{*}{$\begin{array}{l}2019 \text { Flux Estimate } \\
\text { (MMT } \mathrm{CO}_{2} \text { Eq.) }\end{array}$} & \multicolumn{4}{|c|}{ Uncertainty Range Relative to Emission Estimate } \\
\hline & & \multicolumn{2}{|c|}{ (MMT $\mathrm{CO}_{2}$ Eq.) } & \multicolumn{2}{|c|}{$(\%)$} \\
\hline & & $\begin{array}{l}\text { Lower } \\
\text { Bound }\end{array}$ & $\begin{array}{l}\text { Upper } \\
\text { Bound }\end{array}$ & $\begin{array}{l}\text { Lower } \\
\text { Bound }\end{array}$ & $\begin{array}{l}\text { Upper } \\
\text { Bound }\end{array}$ \\
\hline Forest Ecosystem C Pools & $(23.4)$ & $(28.9)$ & $(17.8)$ & $-23.6 \%$ & $23.6 \%$ \\
\hline
\end{tabular}

${ }^{a}$ Range of flux estimates predicted through a combination of sample-based and model-based uncertainty for a 95 percent confidence interval, IPCC Approach 1.

Notes: Parentheses indicate negative values or net uptake. Totals may not sum due to independent rounding.

Table 71: Quantitative Uncertainty Estimates of Non- $\mathrm{CO}_{2}$ Emissions from Forest Fires $\left(\mathrm{MMT} \mathrm{CO}_{2}\right.$ Eq. and Percent), Tennessee $^{\mathrm{a}}$

\begin{tabular}{|c|c|c|c|c|c|c|}
\hline \multirow[b]{2}{*}{ Source } & \multirow[b]{2}{*}{ Gas } & \multirow[b]{2}{*}{$\begin{array}{l}2019 \text { Emission Estimate } \\
\text { (MMT CO2 Eq.) }\end{array}$} & \multicolumn{4}{|c|}{$\begin{array}{l}\text { Uncertainty Range Relative to Emission Estimate } \\
\text { (MMT } \mathrm{CO}_{2} \text { Eq.) }\end{array}$} \\
\hline & & & $\begin{array}{l}\text { Lower } \\
\text { Bound }\end{array}$ & $\begin{array}{l}\text { Upper } \\
\text { Bound }\end{array}$ & $\begin{array}{l}\text { Lower } \\
\text { Bound }\end{array}$ & $\begin{array}{l}\text { Upper } \\
\text { Bound }\end{array}$ \\
\hline Non- $\mathrm{CO}_{2}$ Emissions from Forest Fires & $\mathrm{CH}_{4}$ & + & + & + & $-86.1 \%$ & $138.7 \%$ \\
\hline Non- $\mathrm{CO}_{2}$ Emissions from Forest Fires & $\mathrm{N}_{2} \mathrm{O}$ & + & + & + & $-78.1 \%$ & $115.9 \%$ \\
\hline
\end{tabular}

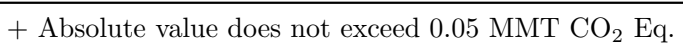

a These estimates include non- $\mathrm{CO}_{2}$ emissions from forest fires on Forest Land Remaining Forest Land and Land Converted to Forest Land.

${ }^{\mathrm{b}}$ Range of flux estimates predicted by Monte Carlo Stochastic Simulation for a 95 percent confidence interval, IPCC Approach 2. 


\section{Texas}

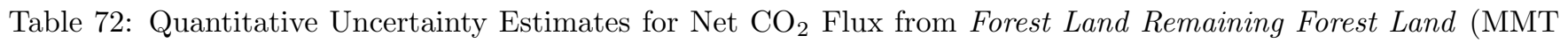
$\mathrm{CO}_{2}$ Eq. and Percent), Texas

\begin{tabular}{|c|c|c|c|c|c|}
\hline \multirow[b]{2}{*}{ Source } & \multirow[b]{2}{*}{$\begin{array}{l}2019 \text { Flux Estimate } \\
\text { (MMT } \mathrm{CO}_{2} \text { Eq.) }\end{array}$} & $\begin{array}{r}\text { Uncert } \\
(\mathrm{MM} \\
\end{array}$ & $\begin{array}{l}\text { Range } R \epsilon \\
\text { Eq.) }\end{array}$ & to Emiss & imate $^{a}$ \\
\hline & & $\begin{array}{l}\text { Lower } \\
\text { Bound }\end{array}$ & $\begin{array}{l}\text { Upper } \\
\text { Bound }\end{array}$ & $\begin{array}{l}\text { Lower } \\
\text { Bound }\end{array}$ & $\begin{array}{l}\text { Upper } \\
\text { Bound }\end{array}$ \\
\hline Forest Ecosystem C Pools & $(20.1)$ & $(22.0)$ & $(18.2)$ & $-9.4 \%$ & $9.4 \%$ \\
\hline
\end{tabular}

${ }^{a}$ Range of flux estimates predicted through a combination of sample-based and model-based uncertainty for a 95 percent confidence interval, IPCC Approach 1.

Notes: Parentheses indicate negative values or net uptake. Totals may not sum due to independent rounding.

Table 73: Quantitative Uncertainty Estimates of Non- $\mathrm{CO}_{2}$ Emissions from Forest Fires $\left(\mathrm{MMT} \mathrm{CO}_{2}\right.$ Eq. and Percent), Texas $^{\mathrm{a}}$

\begin{tabular}{|c|c|c|c|c|c|c|}
\hline \multirow[b]{3}{*}{ Source } & \multirow[b]{3}{*}{ Gas } & \multirow[b]{3}{*}{$\begin{array}{l}2019 \text { Emission Estimate } \\
\text { (MMT CO2 Eq.) }\end{array}$} & \multicolumn{4}{|c|}{ Uncertainty Range Relative to Emission Estimate ${ }^{\mathrm{b}}$} \\
\hline & & & \multicolumn{2}{|c|}{ (MMT CO $\mathrm{CO}_{2}$ Eq.) } & \multicolumn{2}{|c|}{$(\%)$} \\
\hline & & & $\begin{array}{l}\text { Lower } \\
\text { Bound }\end{array}$ & $\begin{array}{l}\text { Upper } \\
\text { Bound }\end{array}$ & $\begin{array}{l}\text { Lower } \\
\text { Bound }\end{array}$ & $\begin{array}{l}\text { Upper } \\
\text { Bound }\end{array}$ \\
\hline Non- $\mathrm{CO}_{2}$ Emissions from Forest Fires & $\mathrm{CH}_{4}$ & 0.1 & 0.1 & 0.1 & $-26.7 \%$ & $30.2 \%$ \\
\hline Non- $\mathrm{CO}_{2}$ Emissions from Forest Fires & $\mathrm{N}_{2} \mathrm{O}$ & 0.1 & + & 0.1 & $-24.1 \%$ & $26.4 \%$ \\
\hline
\end{tabular}

+ Absolute value does not exceed $0.05 \mathrm{MMT} \mathrm{CO}_{2} \mathrm{Eq}$.

a These estimates include non- $\mathrm{CO}_{2}$ emissions from forest fires on Forest Land Remaining Forest Land and Land Converted to Forest Land.

${ }^{\mathrm{b}}$ Range of flux estimates predicted by Monte Carlo Stochastic Simulation for a 95 percent confidence interval, IPCC Approach 2. 


\section{Utah}

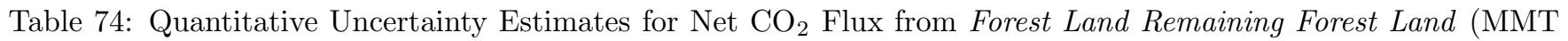
$\mathrm{CO}_{2}$ Eq. and Percent), Utah

\begin{tabular}{|c|c|c|c|c|c|}
\hline \multirow[b]{3}{*}{ Source } & \multirow[b]{3}{*}{$\begin{array}{l}2019 \text { Flux Estimate } \\
\text { (MMT CO } \mathrm{CO}_{2} \text { Eq.) }\end{array}$} & \multicolumn{4}{|c|}{ Uncertainty Range Relative to Emission Estimate ${ }^{a}$} \\
\hline & & \multicolumn{2}{|c|}{ (MMT $\mathrm{CO}_{2}$ Eq.) } & \multicolumn{2}{|c|}{$(\%)$} \\
\hline & & $\begin{array}{l}\text { Lower } \\
\text { Bound }\end{array}$ & $\begin{array}{l}\text { Upper } \\
\text { Bound }\end{array}$ & $\begin{array}{l}\text { Lower } \\
\text { Bound }\end{array}$ & $\begin{array}{l}\text { Upper } \\
\text { Bound }\end{array}$ \\
\hline Forest Ecosystem C Pools & 3.8 & (1.3) & 8.8 & $-134.7 \%$ & $134.7 \%$ \\
\hline
\end{tabular}

Table 75: Quantitative Uncertainty Estimates of Non- $\mathrm{CO}_{2}$ Emissions from Forest Fires $\left(\mathrm{MMT} \mathrm{CO}_{2}\right.$ Eq. and Percent), $\mathrm{Utah}^{\mathrm{a}}$

\begin{tabular}{|c|c|c|c|c|c|c|}
\hline \multirow[b]{2}{*}{ Source } & \multirow[b]{2}{*}{ Gas } & \multirow[b]{2}{*}{$\begin{array}{l}2019 \text { Emission Estimate } \\
\text { (MMT CO2 Eq.) }\end{array}$} & \multicolumn{4}{|c|}{$\begin{array}{l}\text { Uncertainty Range Relative to Emission Estimate } \\
\text { (MMT } \mathrm{CO}_{2} \text { Eq.) }\end{array}$} \\
\hline & & & $\begin{array}{l}\text { Lower } \\
\text { Bound }\end{array}$ & $\begin{array}{l}\text { Upper } \\
\text { Bound }\end{array}$ & $\begin{array}{l}\text { Lower } \\
\text { Bound }\end{array}$ & $\begin{array}{l}\text { Upper } \\
\text { Bound }\end{array}$ \\
\hline Non- $\mathrm{CO}_{2}$ Emissions from Forest Fires & $\mathrm{CH}_{4}$ & 0.7 & 0.4 & 1.0 & $-40.3 \%$ & $49 \%$ \\
\hline Non- $\mathrm{CO}_{2}$ Emissions from Forest Fires & $\mathrm{N}_{2} \mathrm{O}$ & 0.4 & 0.3 & 0.6 & $-31.6 \%$ & $38.6 \%$ \\
\hline
\end{tabular}

a These estimates include non- $\mathrm{CO}_{2}$ emissions from forest fires on Forest Land Remaining Forest Land and Land Converted to Forest Land.

${ }^{\mathrm{b}}$ Range of flux estimates predicted by Monte Carlo Stochastic Simulation for a 95 percent confidence interval, IPCC Approach 2. 


\section{Vermont}

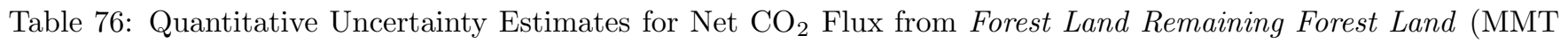
$\mathrm{CO}_{2}$ Eq. and Percent), Vermont

\begin{tabular}{|c|c|c|c|c|c|}
\hline \multirow[b]{3}{*}{ Source } & \multirow[b]{3}{*}{$\begin{array}{l}2019 \text { Flux Estimate } \\
\text { (MMT } \mathrm{CO}_{2} \text { Eq.) }\end{array}$} & \multicolumn{4}{|c|}{ Uncertainty Range Relative to Emission Estimate } \\
\hline & & \multicolumn{2}{|c|}{ (MMT $\mathrm{CO}_{2}$ Eq.) } & \multicolumn{2}{|c|}{$(\%)$} \\
\hline & & $\begin{array}{l}\text { Lower } \\
\text { Bound }\end{array}$ & $\begin{array}{l}\text { Upper } \\
\text { Bound }\end{array}$ & $\begin{array}{l}\text { Lower } \\
\text { Bound }\end{array}$ & $\begin{array}{l}\text { Upper } \\
\text { Bound }\end{array}$ \\
\hline Forest Ecosystem C Pools & $(5.9)$ & $(8.5)$ & $(3.2)$ & $-44.6 \%$ & $44.6 \%$ \\
\hline
\end{tabular}

${ }^{a}$ Range of flux estimates predicted through a combination of sample-based and model-based uncertainty for a 95 percent confidence interval, IPCC Approach 1.

Notes: Parentheses indicate negative values or net uptake. Totals may not sum due to independent rounding. 


\section{Virginia}

Table 77: Quantitative Uncertainty Estimates for Net $\mathrm{CO}_{2}$ Flux from Forest Land Remaining Forest Land (MMT $\mathrm{CO}_{2}$ Eq. and Percent), Virginia

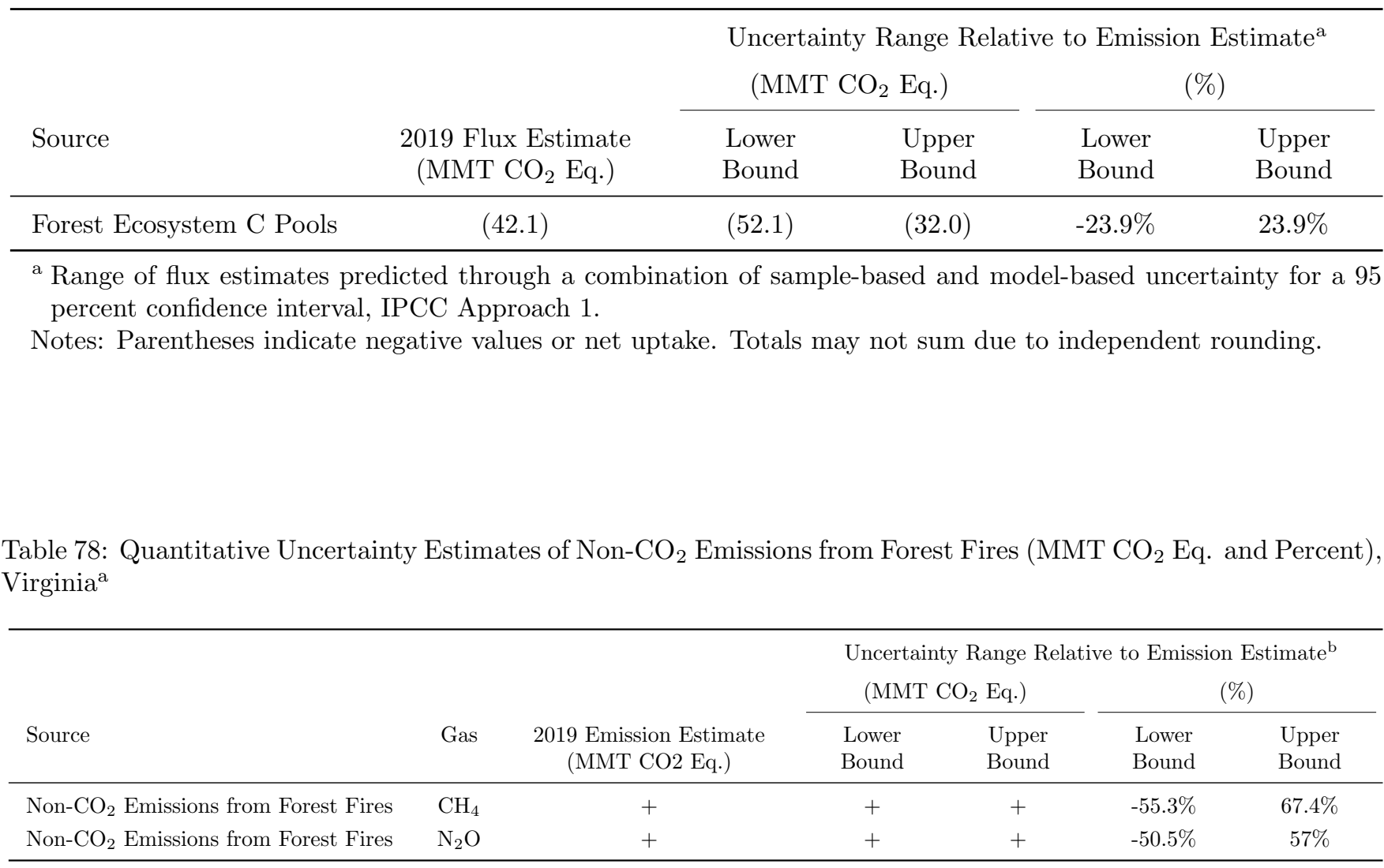

+ Absolute value does not exceed $0.05 \mathrm{MMT} \mathrm{CO}_{2} \mathrm{Eq}$.

a These estimates include non- $\mathrm{CO}_{2}$ emissions from forest fires on Forest Land Remaining Forest Land and Land Converted to Forest Land.

${ }^{\mathrm{b}}$ Range of flux estimates predicted by Monte Carlo Stochastic Simulation for a 95 percent confidence interval, IPCC Approach 2. 


\section{Washington}

Table 79: Quantitative Uncertainty Estimates for Net $\mathrm{CO}_{2}$ Flux from Forest Land Remaining Forest Land (MMT $\mathrm{CO}_{2}$ Eq. and Percent), Washington

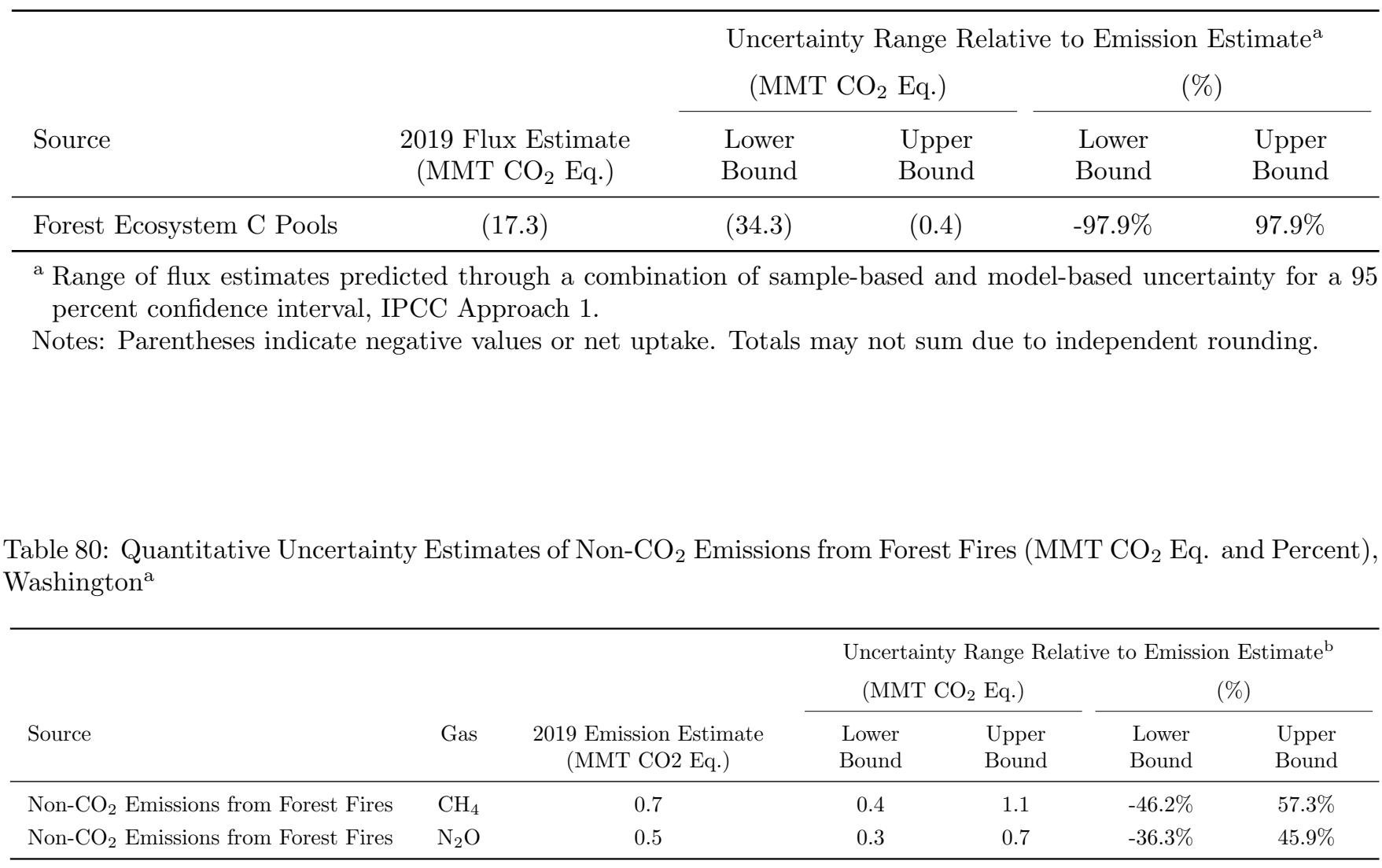

${ }^{a}$ These estimates include non- $\mathrm{CO}_{2}$ emissions from forest fires on Forest Land Remaining Forest Land and Land Converted to Forest Land.

${ }^{\mathrm{b}}$ Range of flux estimates predicted by Monte Carlo Stochastic Simulation for a 95 percent confidence interval, IPCC Approach 2. 


\section{West Virginia}

Table 81: Quantitative Uncertainty Estimates for Net $\mathrm{CO}_{2}$ Flux from Forest Land Remaining Forest Land (MMT $\mathrm{CO}_{2}$ Eq. and Percent), West Virginia

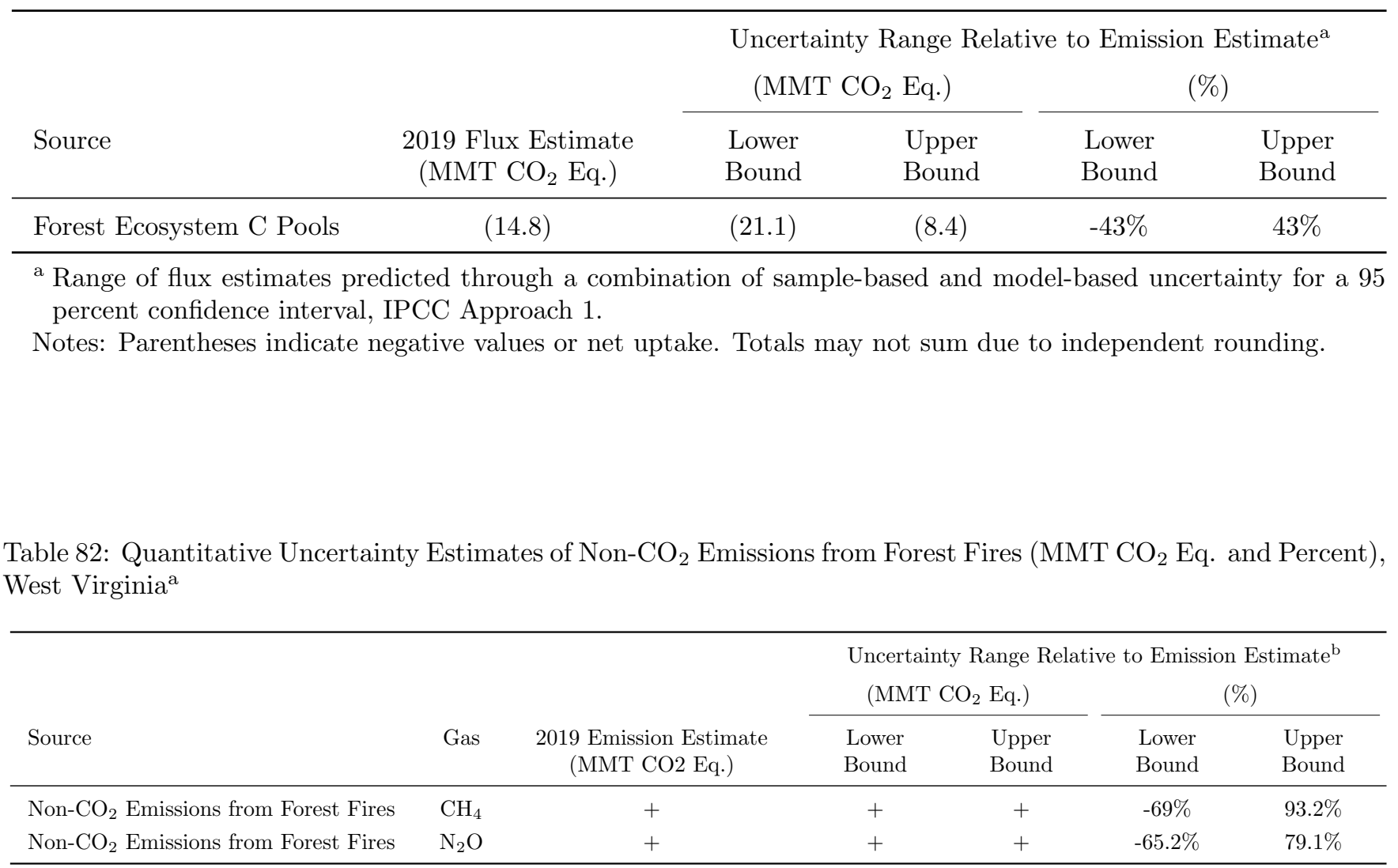

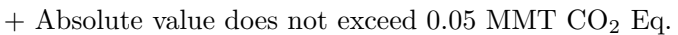

a These estimates include non- $\mathrm{CO}_{2}$ emissions from forest fires on Forest Land Remaining Forest Land and Land Converted to Forest Land.

${ }^{\mathrm{b}}$ Range of flux estimates predicted by Monte Carlo Stochastic Simulation for a 95 percent confidence interval, IPCC Approach 2. 


\section{Wisconsin}

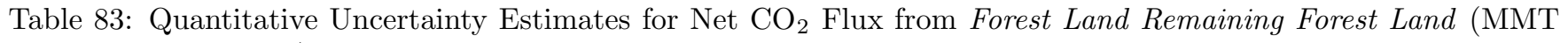
$\mathrm{CO}_{2}$ Eq. and Percent), Wisconsin

\begin{tabular}{|c|c|c|c|c|c|}
\hline \multirow[b]{3}{*}{ Source } & \multirow{3}{*}{$\begin{array}{l}2019 \text { Flux Estimate } \\
\text { (MMT } \mathrm{CO}_{2} \text { Eq.) }\end{array}$} & \multicolumn{4}{|c|}{ Uncertainty Range Relative to Emission Estimate } \\
\hline & & \multicolumn{2}{|c|}{ (MMT CO $\mathrm{CO}_{2}$ Eq.) } & \multicolumn{2}{|c|}{$(\%)$} \\
\hline & & Lower & Upper & Lower & Upper \\
\hline Forest Ecosystem C Pools & $(16.2)$ & $(17.9)$ & $(14.5)$ & $-10.4 \%$ & $10.4 \%$ \\
\hline
\end{tabular}

${ }^{a}$ Range of flux estimates predicted through a combination of sample-based and model-based uncertainty for a 95 percent confidence interval, IPCC Approach 1.

Notes: Parentheses indicate negative values or net uptake. Totals may not sum due to independent rounding. 


\section{Wyoming}

Table 84: Quantitative Uncertainty Estimates for Net $\mathrm{CO}_{2}$ Flux from Forest Land Remaining Forest Land (MMT $\mathrm{CO}_{2}$ Eq. and Percent), Wyoming

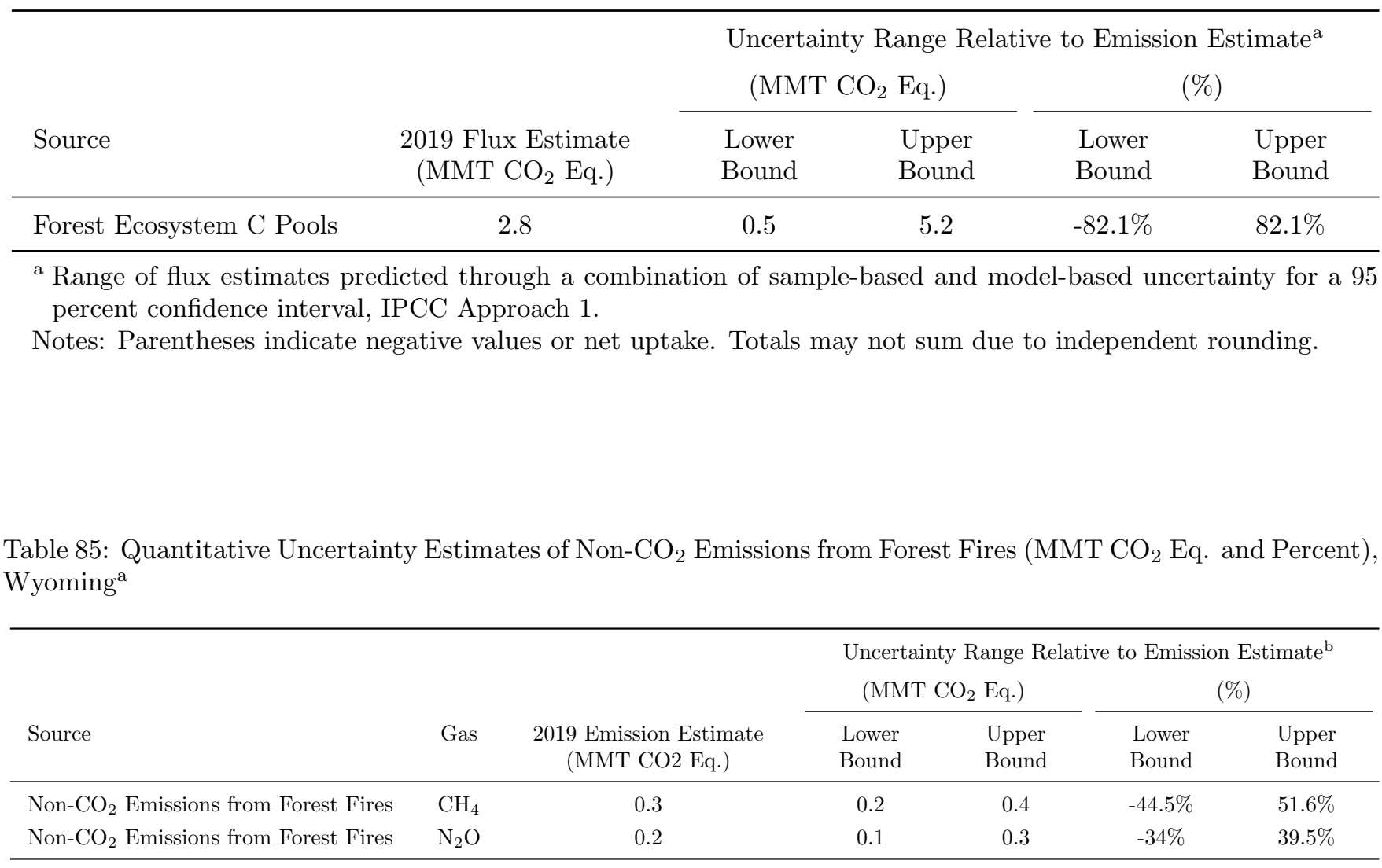

${ }^{a}$ These estimates include non- $\mathrm{CO}_{2}$ emissions from forest fires on Forest Land Remaining Forest Land and Land Converted to Forest Land.

${ }^{\mathrm{b}}$ Range of flux estimates predicted by Monte Carlo Stochastic Simulation for a 95 percent confidence interval, IPCC Approach 2. 